\title{
OPEN Impact of osmotic stress on the phosphorylation and subcellular location of Listeria monocytogenes stressosome proteins
}

Charlotte Dessaux ${ }^{1}$, Duarte N. Guerreiro ${ }^{2}$, M. Graciela Pucciarelli ${ }^{1,3}$, Conor P. O’Byrne ${ }^{2}$ \& Francisco García-del Portillo ${ }^{1 凶}$

Listeria monocytogenes responds to environmental stress using a supra-macromolecular complex, the stressosome, to activate the stress sigma factor SigB. The stressosome structure, inferred from in vitro-assembled complexes, consists of the core proteins RsbR (here renamed RsbR1) and RsbS and, the kinase RsbT. The active complex is proposed to be tethered to the membrane and to support RsbR1/RsbS phosphorylation by RsbT and the subsequent release of RsbT following signal perception. Here, we show in actively-growing cells that $L$. monocytogenes RsbR1 and RsbS localize mostly in the cytosol in a fully phosphorylated state regardless of osmotic stress. RsbT however distributes between cytosolic and membrane-associated pools. The kinase activity of RsbT on RsbR1/RsbS and its requirement for maximal SigB activation in response to osmotic stress were demonstrated in vivo. Cytosolic RsbR1 interacts with RsbT, while this interaction diminishes at the membrane when RsbR1 paralogues (RsbR2, RsbR3 and RsbL) are present. Altogether, the data support a model in which phosphorylated RsbR1/RsbS may sustain basal SigB activity in unstressed cells, probably assuring a rapid increase in such activity in response to stress. Our findings also suggest that in vivo the active RsbR1-RsbS-RsbT complex forms only transiently and that membrane-associated RsbR1 paralogues could modulate its assembly.

Stress response is central for all organisms to adapt and survive in adverse environmental conditions. Grampositive bacteria like Bacillus subtilis and Listeria monocytogenes use an alternative sigma factor, named SigB $\left(\sigma^{\mathrm{B}}\right)$, that binds to the RNA polymerase core to drive the stress response by altering expression of up to 300 genes $^{1-5}$. SigB activity is controlled by an anti-sigma factor, RsbW ${ }^{6}$. Bacteria adjust the amount of RsbW capable of sequestering SigB using the anti-anti-sigma factor RsbV. RsbW can associate preferentially with RsbV when the latter is dephosphorylated through the action of RsbU, a specific phosphatase. The RsbV:RsbV $\sim \mathrm{P}$ ratio therefore determines the amount of free SigB that is available to associate with the core RNA polymerase and, as consequence, the intensity of the transcriptional output response ${ }^{7,8}$. In B. subtilis, RsbV phosphorylation levels can be modulated by either RsbU or RsbP phosphatases depending on the type of signal perceived whether it is environmental or energy stress, respectively ${ }^{9-11}$. Listeria monocytogenes lacks the RsbP-like phosphatase and its RsbQ cofactor found in B. subtilis, which indicates that, in this bacterium, the cascades responding to environmental and energy stresses occur in a single RsbU-dependent pathway ${ }^{12}$ (Fig. 1).

The integration of environmental stress signals was first proposed in B. subtilis and, later confirmed in $L$. monocytogenes, to rely on a supra-macromolecular complex named the stressosome. This complex was assembled in vitro using purified recombinant proteins ${ }^{13-16}$. These experiments established the key structural roles of two core proteins: RsbS and RsbR, this latter known as RsbRA in B. subtilis and, proposed here to be renamed RsbR1 for the L. monocytogenes paralogue. These two core proteins are hypothesised to be phosphorylated by

${ }^{1}$ Laboratory of Intracellular Bacterial Pathogens, National Centre for Biotechnology (CNB)-CSIC, Darwin 3, 28049 Madrid, Spain. ${ }^{2}$ Bacterial Stress Response Group, Microbiology, School of Natural Sciences, National University of Ireland Galway, Galway H91 TK33, Ireland. ${ }^{3}$ Department of Molecular Biology, Centre of Molecular Biology 'Severo Ochoa' (CBMSO)-CSIC, Universidad Autónoma de Madrid, 28049 Madrid, Spain. ${ }^{\square}$ email: fgportillo@cnb.csic.es 


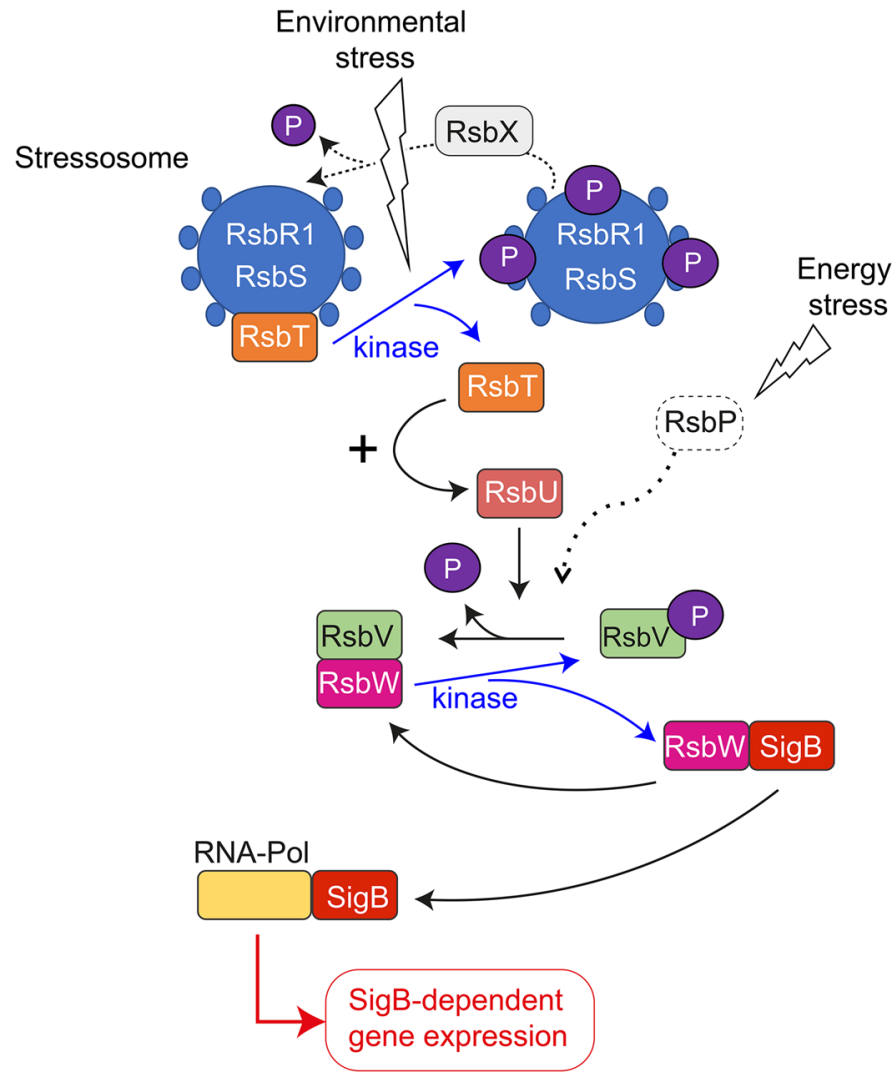

Figure 1. Model of the regulatory signalling cascade that controls SigB activity. The model highlights the role of RsbV phosphorylation in controlling SigB activity, through its modulation of the levels of free RsbW, the anti-sigma factor that sequesters SigB. RsbT is released from the stressosome following activation of its kinase activity in response to stress signals and this promotes its association with RsbU, which in turn promotes the dephosphorylation of RsbV and subsequent release of SigB from RsbW. The scheme is based mostly in data obtained in B. subtilis, including the alternative stimulatory pathway that responds to energy stress and involves $\mathrm{RsbP}$, a protein that is absent in L. monocytogenes. The putative role of RsbX as phosphatase reverting the stimulatory state of the stressosome (with RsbT released from the complex) is also depicted. RNA-Pol, RNA polymerase. See text for further details.

the kinase RsbT following the detection of a stress sensory input. Recent in vitro studies performed with purified L. monocytogenes proteins showed that the RsbR1-RsbS-RsbT complex, as visualized by negative-stain electron microscopy, assembles as a more homogeneous particle than the RsbR1-RsbS complex ${ }^{16}$. The atomic structure of the RsbR1-RsbS-RsbT complex, as determined by cryogenic electron microscopy, reveals an icosahedronlike architecture with sensory domains protruding from the surface (turrets), produced by 20 RsbR1-dimers interacting with 10 RsbS dimers and 10 RsbT dimers ${ }^{16}$. The widely accepted model in both B. subtilis and $L$. monocytogenes involves assembly of RsbR1 and RsbS in a mono- and unphosphorylated stage, respectively ${ }^{16,17}$. The current model predicts that stress signal perception stimulates the kinase activity of RsbT to phosphorylate the second site in RsbR1 and the single site present in $\mathrm{RsbS}^{18-22}$. These phosphorylation events cause the release of RsbT from the stressosome complex and allow its interaction with RsbU, leading to the dephosphorylation of RsbV $\sim$ P, sequestration of RsbW and thereby SigB activation ${ }^{10,23,24}$. Most of these steps have been inferred from in vitro studies based on recombinant proteins lacking kinase or phosphatase activities. For example, in one study in B. subtilis an RsbT variant lacking its kinase activity was unable to form a complex together with RsbRA and RsbS ${ }^{25}$.

Recent studies in L. monocytogenes have also uncovered the importance of the subcellular location of the stressosome to properly integrate the external stress signal. An $\mathrm{N}$-terminomics analysis in L. monocytogenes identified a membrane spanning miniprotein, Prli42, which was proposed to tether the stressosome complex to the membrane in response to oxidative stress by interacting with RsbR $1^{15}$. Interestingly, immunoprecipitation assays shown in that study indicated that Prli42 also interacts with three RsbR1 paralogue proteins, Lmo0161, Lmo0799 and Lmo1642, with a fourth paralogue, Lmo1842, not detected in these assays. Lmo0799 was recently renamed as RsbL ${ }^{26}$ to reflect its involvement in light sensing. We propose here to rename Lmo0161, Lmo1642 and Lmo1842 as RsbR2, RsbR3 and RsbR4, respectively. RsbR2, RsbR3, RbsR4 and RsbL are orthologues of the B. subtilis proteins RsbRB, RsbRC, RsbRD, and YtvA, respectively. Although not specifically discussed in the study of Impens et al. in L. monocytogenes ${ }^{15}$, the immunoprecipitation of RsbR1 paralogues with the miniprotein Prli42 opens the possibility of heterogeneous stressosome complexes associating with the plasma membrane. 
Even though the core stressosome of L. monocytogenes clearly comprises RsbR1, RsbS and RsbT, it is not yet clear how the in vivo structure of the stressosome varies over time and space, particularly in relation to the relative abundance of the RsbR1 paralogues.

Despite the information obtained from structural analyses and the protein-protein interactions characterized in vitro within the RsbR1-RsbS-RsbT complex, there remains much to learn about the dynamics of these interactions and how they are modulated by live cells in response to stress. Here, we have addressed in actively growing $L$. monocytogenes cells the subcellular location of the main stressosome components, their interactions and the phosphorylation status in the absence/presence of osmotic stress. Unexpectedly, some of these proteins were detected mostly in the cytosol and with phosphorylation states that remained unaltered in response to hyperosmolarity. Our findings also suggest that some of the RsbR1 paralogues may negatively modulate the functioning of the stressosome in vivo.

\section{Results}

RsbR1 and RsbS are mainly cytosolic in L. monocytogenes live cells regardless of osmotic stress. Recent studies performed in L. monocytogenes show that RsbR (hereinafter renamed as RsbR1) and RsbS are core proteins of the stressosome complex, which can be tethered to the membrane by the miniprotein Prli42 for the response to oxidative stress ${ }^{15,16}$. However, the distribution of these core proteins or the kinase RsbT between cytosol and membrane has been addressed exclusively for RsbR $1^{15}$. To assess the relative levels of the stressosome components at each subcellular location in vivo, we prepared cytosol and membrane fractions of L. monocytogenes wild-type strain EGD-e growing exponentially in the nutrient-rich medium BHI. A culture of bacteria exposed for $30 \mathrm{~min}$ to $\mathrm{BHI}$ supplemented with $0.5 \mathrm{M} \mathrm{NaCl}$ was run in parallel to determine the effect of osmotic stress on the location or levels of these proteins. Exposure to this salt concentration of $0.5 \mathrm{M} \mathrm{NaCl}$ did not alter L. monocytogenes viability or morphology (Supplementary Fig. S1). By monitoring expression of a highly SigB-dependent gene such as $l m o 2230$ using $a \mathrm{P}_{\text {lmo2230:: }}$ egfp fluorescent reporter fusion, we confirmed that the $30 \mathrm{~min}$ exposure to $0.5 \mathrm{M} \mathrm{NaCl}$ is sufficient to activate SigB (Fig. $2 \mathrm{~A}, \mathrm{~B})^{27,28}$. Importantly, we also observed a noticeable basal activity of SigB in unstressed cells using this reporter. Thus, the cytometry assays revealed that $\sim 75 \%$ of the bacterial population displayed a positive signal for eGFP above the background that increased to $\sim 95 \%$ in the population exposed to salt stress (Fig. 2 C). These findings are consistent with a previous study reporting basal SigB activity in unstressed L. monocytogenes cells ${ }^{29}$.

The immunoblot analyses to detect RsbR1, RsbS and RsbT in the subcellular fractions were carried out using affinity-purified antibodies. An important control included in these experiments was a polar $\Delta r s b R 1$ mutant known to have affected the expression of the downstream genes $r s b S$ and $r s b T^{30}$. These assays showed that most of the RsbR1 and RsbS were in cytosol, regardless of presence or absence of osmotic stress (Fig. 3A-C). Although we consistently failed to detect RsbS by immunoblot in membrane fractions (Fig. 3C), RsbR1 was however visualized in highly concentrated (tenfold) membrane fractions (Fig. 3A). Control immunoblots against purified RsbR1 and RsbS showed that the antibodies have equal affinity (Supplementary Fig. S2). Unlike RsbS, the kinase RsbT was however easily detected in both the cytosolic and membrane fractions (Fig. 3D). No alterations in the amount of RsbT present in cytosol and membrane were observed in response to osmotic stress.

Similar to RsbT, we unexpectedly detected SigB in both cytosolic and membrane fractions (Fig. 3E). The possibility of cross-contamination between subcellular fractions was discarded as immunoblot assays showed that the abundant chaperone protein GroEL was present exclusively in the cytosol (Fig. 3F). Unlike the three stressosome proteins tested, SigB protein levels clearly increased in response to osmotic stress in both the cytosol and membrane fractions (Fig. $3 \mathrm{E}$ ). Such increased SigB levels agree with the positive autoregulation that SigB exerts over the $r s b V$-rs $b W$-sigB-rs $b X$ operon in stressed bacteria ${ }^{31}$. The unexpected membrane localisation of SigB led us to test whether it was facilitated by transertion of SigB-regulated proteins. To investigate this, we inhibited protein synthesis concomitantly with the increase in osmolarity. Under these conditions, lower levels of membrane-associated SigB were detected relative to the untreated control samples (Supplementary Fig. S3). This finding suggests that some protein(s) upregulated by SigB upon stress could favour membrane localisation of this sigma factor.

Lastly, we sought to determine the distribution of RsbR1, RsbS and RsbT in the absence of the RsbR1 paralogues. To do this, we analysed a L. monocytogenes mutant that lacks the RsbR1 paralogues Lmo0161, Lmo1642 and Lmo1842-here renamed as RsbR2, RsbR3 and RsbR4, respectively- and bears an additional loss-of-function mutation (C56A) in the fourth paralogue RsbL ${ }^{26}$. For simplicity, this quadruple mutant (with the genotype $\Delta \mathrm{rs} b R 2, \Delta r s b R 3, \Delta r s b R 4, r s b L-C 56 A)$ is described hereinafter as " $\Delta 4$ ". The lack of functional RsbR1 paralogues resulted in increased RsbS levels in the cytosol, an effect not observed for the other proteins tested, RsbR1, RsbT or SigB (Fig. 3A-E). Taken together, these data pointed to a predominant localisation of L. monocytogenes stressosome proteins to the cytosol, irrespective of the presence or absence of osmotic stress and increased amounts of $\mathrm{RsbS}$ in the cytosol of bacteria having no functional RsbR1 paralogues.

Listeria monocytogenes RsbR1 and RsbS are predominantly phosphorylated regardless of stress. The current models of stressosome activation in B. subtilis and L. monocytogenes involve phosphorylation of RsbRA (RsbR1) and RsbS by the kinase RsbT concomitant with the exposure to stress ${ }^{16,17}$. L. monocytogenes RsbR1 has two conserved phosphorylatable threonine residues (T175, T209) with one of them, $\mathrm{T} 175$, being phosphorylated in basal non-stressed conditions $\mathrm{s}^{32}$ and reported to be required to respond to acidic stress $^{33}$. RsbS has one conserved phosphorylatable serine residue (S56), proposed to be phosphorylated only upon stress ${ }^{15,18,22}$. Despite L. monocytogenes having the conserved phosphorylatable residues in RsbR1 and RsbS, as in the respective $B$. subtilis orthologues, their phosphorylation dynamics in vivo remains unknown. Only a phosphoproteomic study, performed in unstressed L. monocytogenes cells, reported the identification of a RsbR1 
A plmo2230::egfp

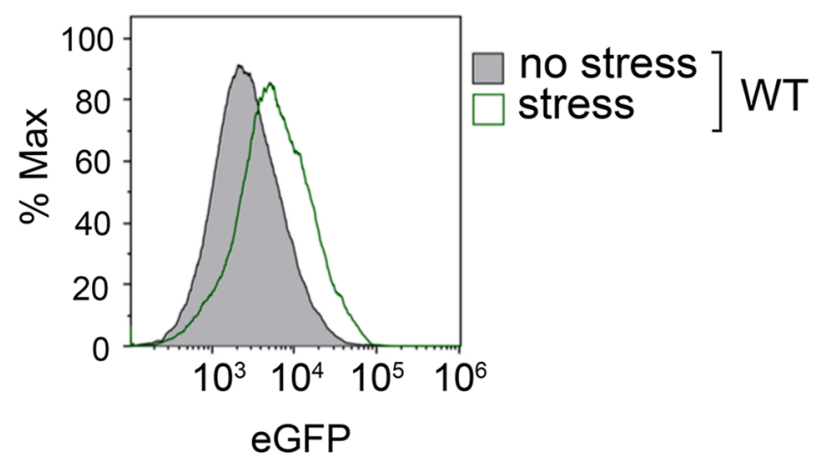

C

\section{no reporter}

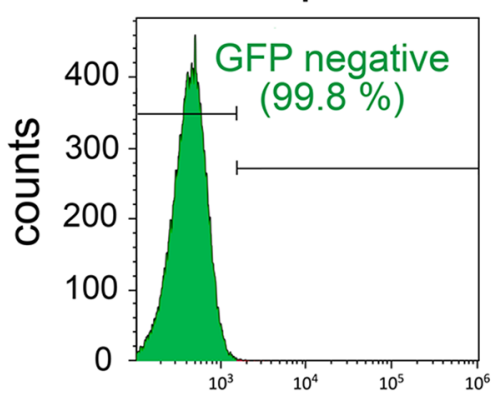

plmo2230::egfp

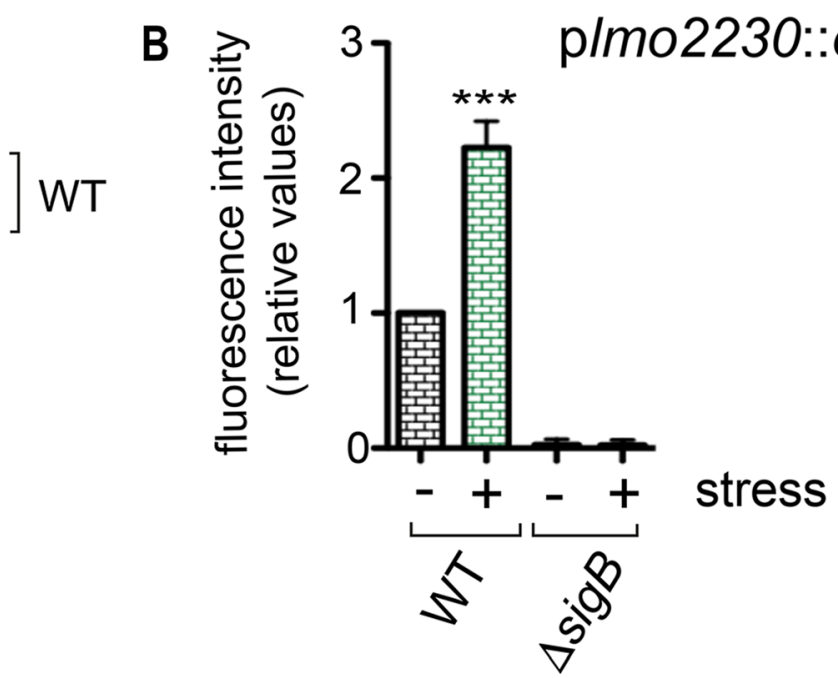

\section{plmo2230::egfo}

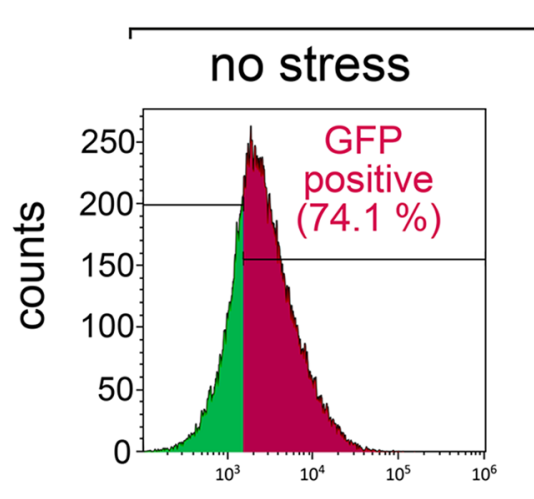

Figure 2. Monitoring of the L. monocytogenes response to osmotic stress with the $\mathrm{P}_{l m o 2230}:$ :egfp reporter fusion.

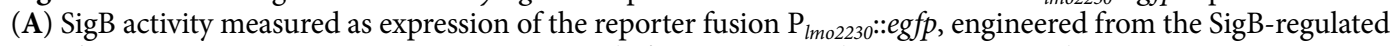
gene lmo2230. Reporter expression was measured after $30 \mathrm{~min}$ incubation in BHI medium containing $0.5 \mathrm{M}$ $\mathrm{NaCl}$. Shown are data representative of a total of three biological replicates. (B) Mean fluorescence intensity quantified in wild-type and DsigB strains in unstress and stress conditions. ${ }^{* *}, P<0.0005$ (one-way ANOVA with Bonferroni's multiple comparisons test). (C) Quantification of the proportion of the bacterial population positive for eGFP levels in the indicated conditions.

peptide containing the phosphorylated T175 P residue ${ }^{32}$. However, no further studies on the kinase involved or the changes occurring under stress, were reported.

To determine in vivo the phosphorylation dynamics of the L. monocytogenes stressosome proteins, we used two types of mutants: one harbouring a predicted kinase-inactive RsbT-N49A variant and, the other expressing a RsbR1-T175A variant, therefore not phosphorylatable at residue 175. To differentiate the different phosphorylated isoforms, we exploited the Phos-Tag system based on phosphate- $\mathrm{Zn}^{2+}$ ion sequestration that results in distinct electrophoretic mobility for the distinct isoforms (see "Methods"). To avoid any putative undesirable phosphorylation event due to stress during sample processing (for example SigB activation by centrifugation is known to occur ${ }^{34}$ ) the bacterial cultures were in all assays heat-inactivated at the time of collection and azide added before the first centrifugation step. In other controls, bacteria were fixed with paraformaldehyde before cell disruption. No changes in the phosphorylation patterns were observed when applying this additional control (Supplementary Fig. 4).

Subcellular fractions were then prepared from L. monocytogenes cells growing exponentially in BHI medium in the absence or presence of $0.5 \mathrm{M} \mathrm{NaCl}$. The Phos-Tag method revealed that most RsbR1 present in the cytosol is doubly phosphorylated, with only $\sim 5 \%$ of the total accounting for the mono-phosphorylated isoform (Fig. 4A). Surprisingly, densitometry of the bands detected in the Western blots showed that the osmotic stress has no effect on the phosphorylation pattern of cytosolic RsbR1, with no switch of the mono-phosphorylated pool to the doubly phosphorylated one in stressed cells (Fig. 4A). Conservation of the phosphorylation pattern following osmotic stress also occurred for membrane-associated RsbR1, detected in this location when using more concentrated membrane samples (10×) (Fig. 4A). This membrane RsbR1 pool was estimated in 5\% of the amount detected in the cytosol. Importantly, membrane-associated RsbR1 was also predominantly in the double-phosphorylated form (Fig. 4A). Consistent with the high level of phosphorylation found for RsbR1, even 
A

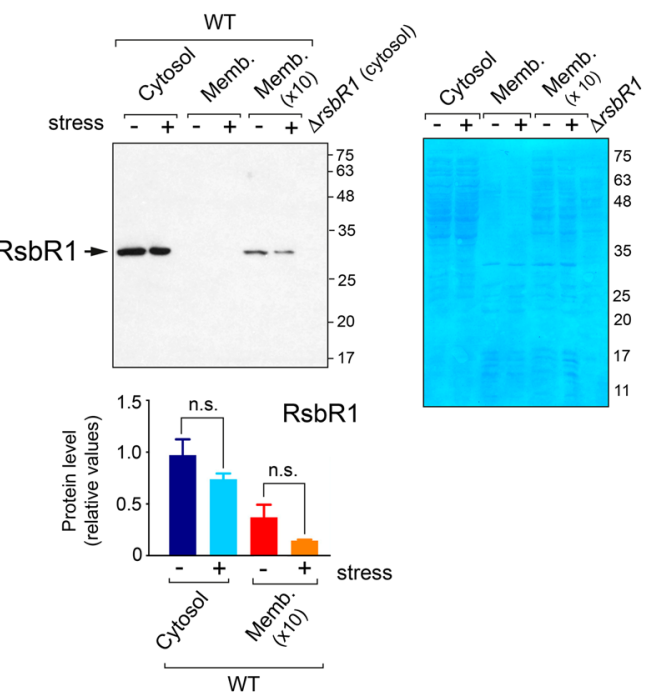

D

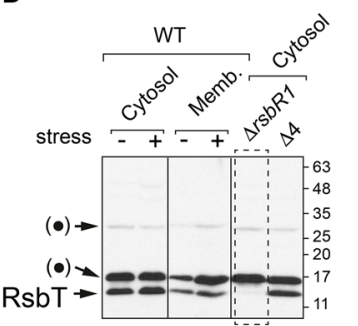

B
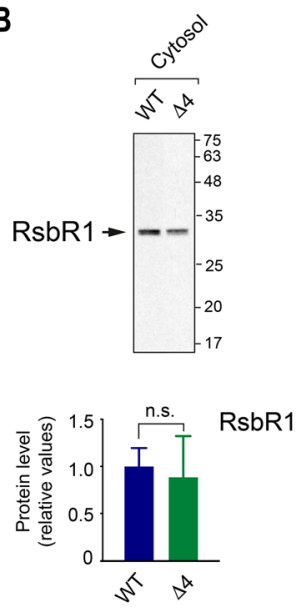

E

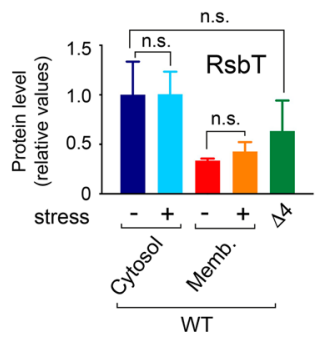

C
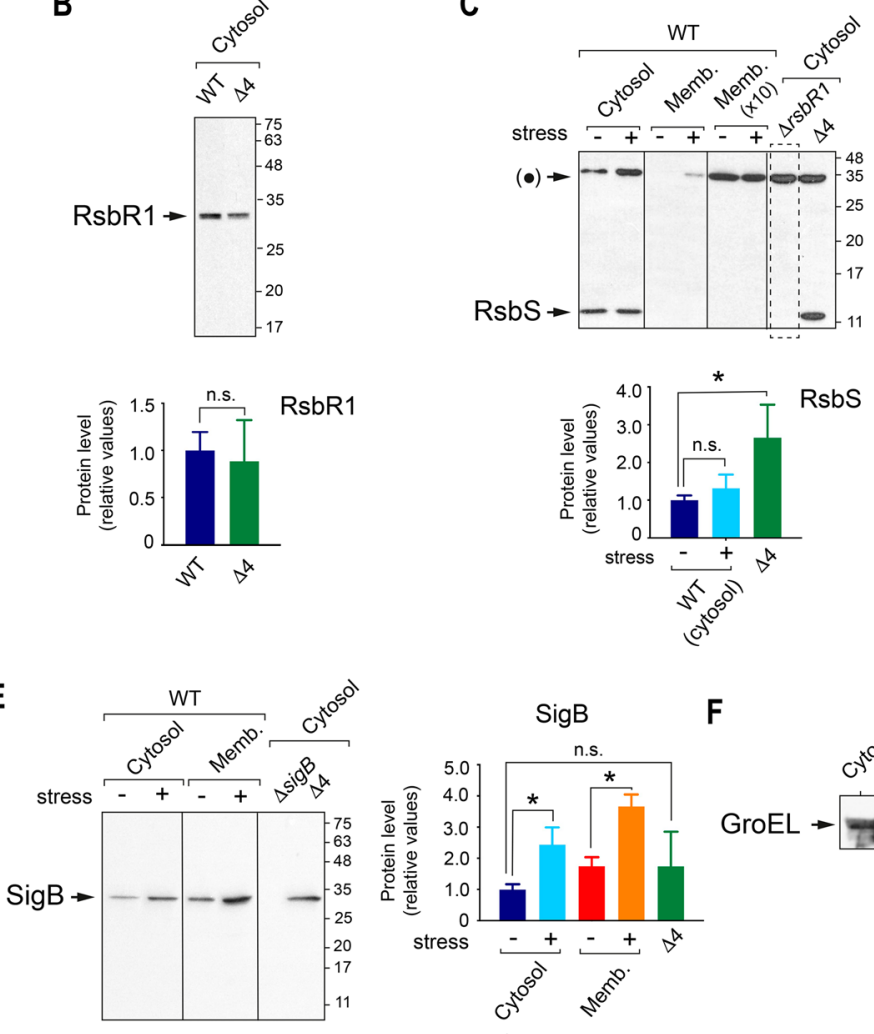

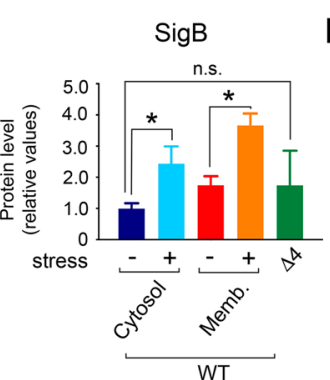

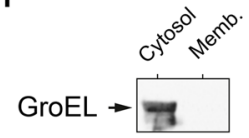

Figure 3. The stressosome proteins RsbR1, RsbS and RsbT abound in the cytosol of L. monocytogenes strain EGD-e, regardless of osmotic stress. Distribution of RsbR1 (A,B), RsbS (C), RsbT (D), and SigB (E) in cytosol and membrane fractions of L. monocytogenes wild type strain EGD-e and derivative strains including a polar $\Delta r s b R 1$ mutant, not expressing RsbR1, RsbS and RsbT; a $\Delta 4$ mutant lacking function of all four RsbR1 paralogues known (see text for details); and a $\triangle \operatorname{sig} B$ mutant. Cytosol and membrane extracts were prepared from unstressed and stressed (exposed $30 \mathrm{~min}$ to $0.5 \mathrm{M} \mathrm{NaCl}$ ) bacteria. Panel A includes a a membrane corresponding to the anti-RsbR1 western blot that was subsequently stained with Coomassie dye. This procedure was used for every blot to verify the proper adjustment of the samples. Quantification of the signals obtained in the blots from three independent biological replicates and for each of the stressosome proteins, is also shown as mean values and standard deviation. ${ }^{*}, P<0.05$; n.s. not significant (one-way ANOVA with Tukey's multiple comparisons test). (F) Control immunoblot with anti-GroEL antibodies discarding crosscontamination between cytosolic and membrane fractions. Bands indicated as $(\bullet)$ in panels $(\mathbf{C , D})$ correspond to unspecific protein recognition. Molecular weight markers are indicated at the right side of each image. Vertical lines within blots indicate separate parts that were grouped from different positions of the same blot. Dashed rectangles highlight the gel lanes corresponding to negative control strains. See Supplementary Fig. S6 for images of full blots.

in the basal unstressed condition, the RsbR1-T175A variant was detected in the mono-phosphorylated state, presumably phosphorylated at residue T209 (Fig. 4A). In the kinase-inactive RsbT-N49A mutant, the entire RsbR1 pool was detected unphosphorylated (Fig. 4A), providing, to our knowledge, the first in vivo evidence for the kinase activity of L. monocytogenes RsbT over its substrate RsbR1.

We next evaluated whether the high phosphorylation rate observed in L. monocytogenes RsbR1 was also noticeable in its B. subtilis paralogue RsbRA. We prepared cytosolic and membrane fractions of B. subtilis growing exponentially in $\mathrm{LB}$ medium and the absence/presence of $0.5 \mathrm{M} \mathrm{NaCl}$. These fractions were analysed for the presence of RsbRA using the Phos-Tag system. Unlike the case in L. monocytogenes, the predominant isoform detected for B. subtilis RsbRA was the mono-phosphorylated, in both the cytosol and the membrane fractions (Fig. 4B). In contrast to the most accepted model of stressosome function, no double-phosphorylated RsbRA was detected in the B. subtilis membrane, even when cells were subjected to osmotic stress conditions (Fig. 4B). The data obtained with B. subtilis, in which the monophosphorylated form predominated, provided an additional control for the lack of phosphorylation events taking place after bacteria were collected for sample processing. The Phos-Tag system also showed that L. monocytogenes RsbS is fully (mono)-phosphorylated, irrespective of stress. This evidence was reached after comparing the electrophoretic mobility of RsbS in the wild-type versus the $r s b T$-N49A kinase-inactive mutant (Fig. 4C). The levels of RsbS were also higher in the rsbR1-T175A mutant respect wild type bacteria (Fig. 4C). Altogether, these data confirmed a high basal kinase activity of L. monocytogenes RsbT in both the cytosol and the membrane over its substrates RsbR1 and RsbS, irrespective of the prevailing osmotic stress conditions. 
A
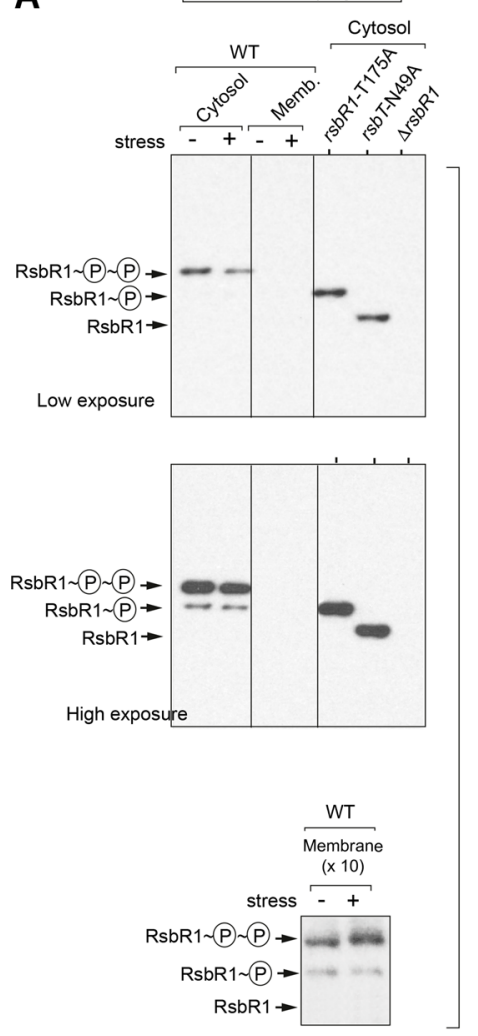

B
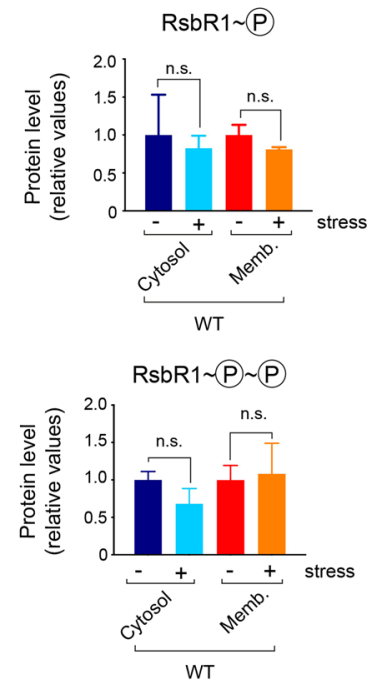
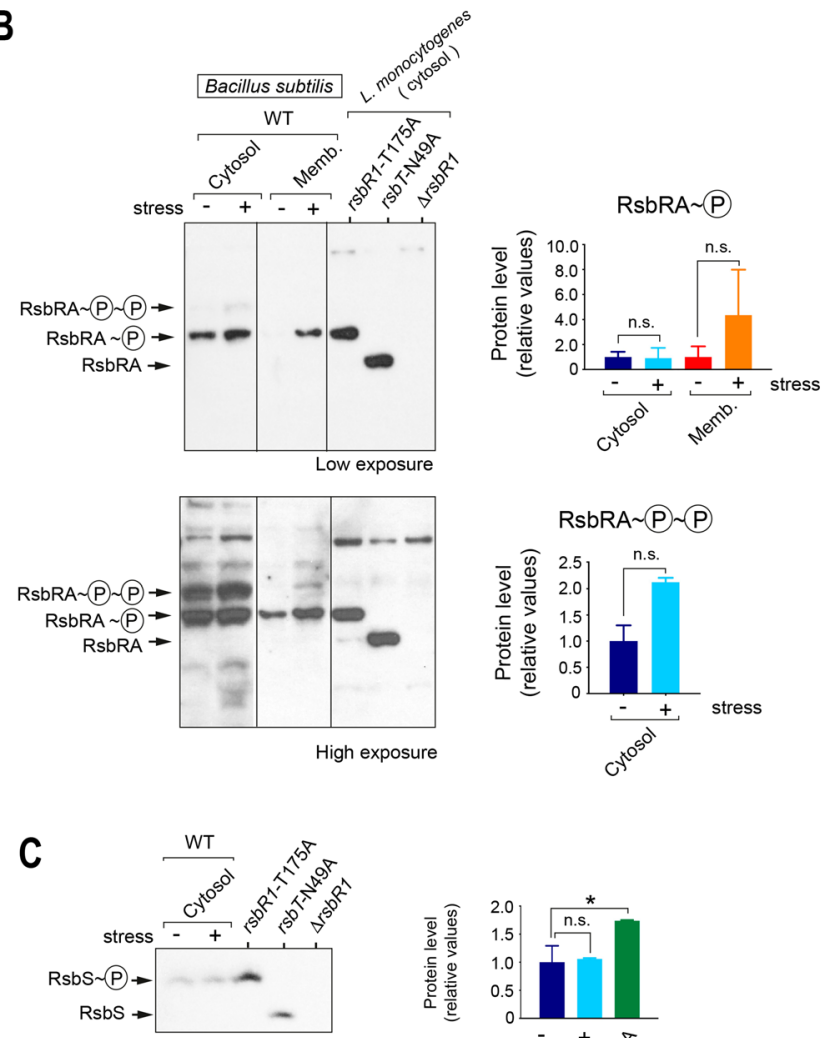

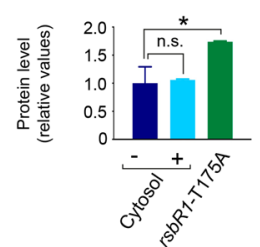

Figure 4. RsbR1 is phosphorylated at large extent by L. monocytogenes, regardless of osmotic stress. (A) Distinct phosphorylated forms of RsbR1 identified by the Phos-Tag system in L. monocytogenes EGD-e subcellular extracts -cytosol/membrane- and in response to $0.5 \mathrm{M} \mathrm{NaCl}$ osmolarity stress. Control samples from strains with the phosphorylatable site T175 mutated (T175A) or with a non-functional RsbT kinase (N49A variant), are included. Note the predominance of the doubly-phosphorylated RsbR1 form in the cytosol and the no detection of membrane-bound RsbR1 with this number of bacteria $\left(5 \times 10^{7}\right)$. Two different exposition times of the western blot are shown. Phosphorylated RsbR1 was detected in membrane fractions of more concentrated membrane samples (10×), corresponding to $5 \times 10^{8}$ bacteria. Note the similar pattern obtained in these membrane fractions compared to the cytosolic fractions. (B) Phosphorylation pattern of B. subtilis RsbRA exposed to the $0.5 \mathrm{M} \mathrm{NaCl}$ osmolarity stress. Two different exposition times are shown. (C) Phosphorylation pattern of L. monocytogenes RsbS in absence or presence of stress. Strains lacking a functional RsbT kinase (N49A) and the polar $\Delta r s b R 1$ mutant were included as controls. Quantification of the signals obtained in three independent biological replicates is shown as mean and standard deviation of a total of three biological replicates. ${ }^{\star} P<0.05$; n.s.: not significant (one-way ANOVA with Tukey’s multiple comparisons test). Vertical lines within blots indicate separate parts that were grouped from different positions of the same blot. See Supplementary Fig. S6 for images of full blots.

RsbR1 phosphorylation and RsbR1 paralogues act antagonistically in SigB activation. Knowing the distinct phosphorylation pattern of RsbR1 in wild-type L. monocytogenes compared to those of the $r s b R 1$-T175A and $r s b T$-N49A mutant strains (Fig. 4), we next sought to determine whether SigB activity correlates with the phosphorylation status of the stressosome proteins. To assess this, we introduced the $\mathrm{P}_{\text {lmo2230 }}:$ :egfp reporter fusion in a series of isogenic wild-type, $\Delta s i g B, r s b R 1$-T175A, $r s b T$-N49A and $\Delta 4$ mutant strains, this latter strain lacking functional RsbR1 paralogues ${ }^{29}$. Cytometry assays allowed us to monitor SigB activity at the population level (Fig. 5A,B). The kinase-inactive $r s b T$-N49A mutant exhibited residual SigB activity above the $\triangle$ sigB mutant but had lost responsiveness to the osmotic stress (Fig. 5A, C). In contrast, the $r s b R 1$-T175A mutant was able to respond to salt stress with increased SigB activity, although not reaching the level detected in wild-type bacteria (Fig. 5B,C). This result highlighted the need for RsbT-mediated phosphorylation to transduce osmotic stress signals but suggested that RsbR1-T175 was partially dispensable for signal transduction, in contrast to previous observations reported for the response to acid stress ${ }^{33}$.

Interestingly, unlike the case of the $r s b R 1-\mathrm{T} 175 \mathrm{~A}$ and $r s b T$-N $49 \mathrm{~A}$ mutants, we detected slightly increased SigB activity in the $\Delta 4$ mutant compared to wild type bacteria in the absence or presence of stress (Fig. $5 \mathrm{~B}, \mathrm{C}$ ), changes that were statistically significant when measuring mean fluorescence intensity of the bacterial populations (Fig. 5C). These observations suggested that RsbR1 has the capacity to form a functional stressosome 
A

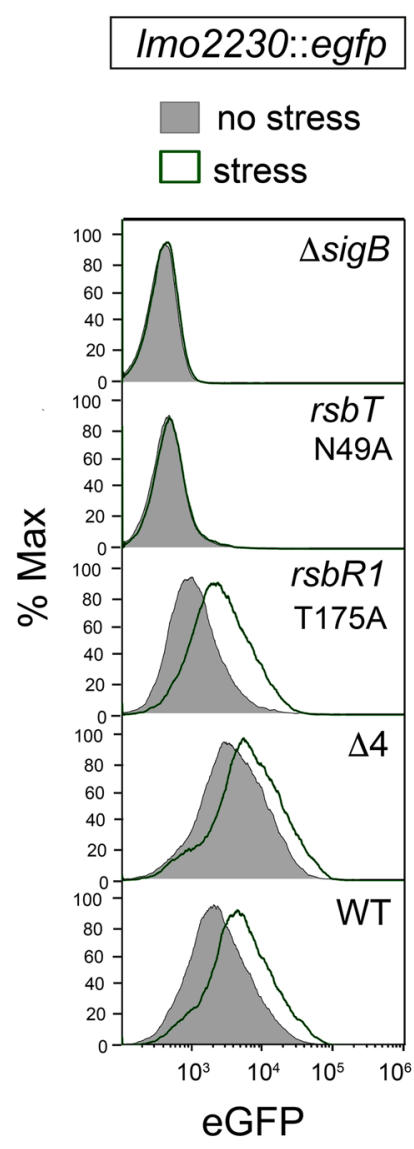

B
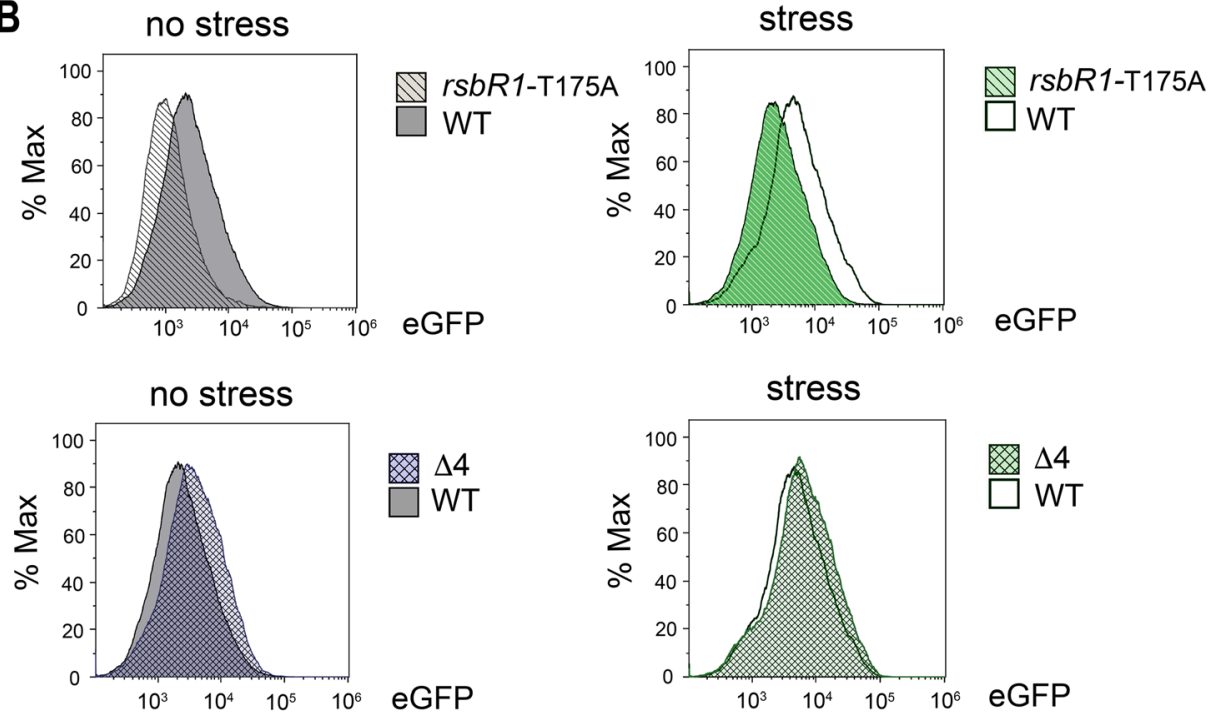

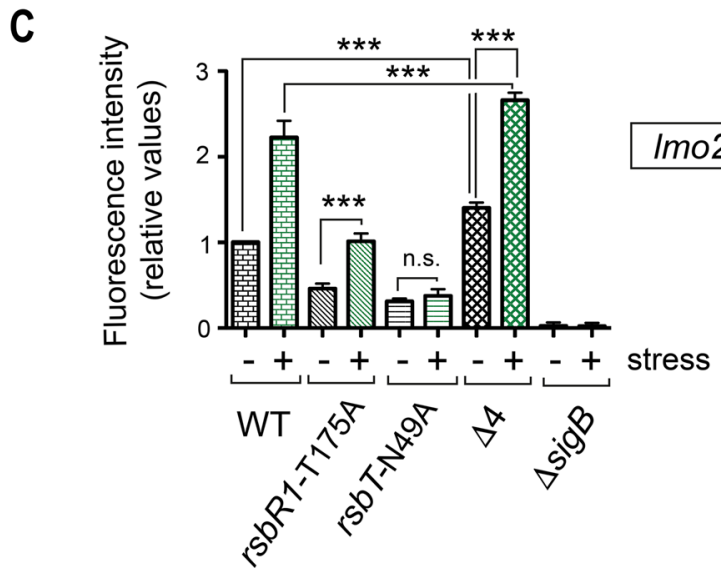

Figure 5. The kinase activity of RsbT is essential for SigB activation with RsbR1 paralogues antagonising such activity in unstressed and stressed L. monocytogenes. (A) Expression of the $\mathrm{P}_{\text {lmo2230: }}$ egfp reporter fusion as proxy of SigB activity in the indicated strains in no stress and stress $(30 \mathrm{~min}, 0.5 \mathrm{M} \mathrm{NaCl})$ conditions. Shown is the fluorescence intensity registered in the bacterial population by flow cytometry. (B) Side-to-side comparison of SigB activity in wild type compared to the mutant lacking the first phosphorylatable threonine residue, T175, and the $\Delta 4$ mutant lacking functional RsbR1 paralogues. Data shown in panels $(\mathbf{A}, \mathbf{B})$ are representative of a total of six samples (3 biological replicates with two technical replicates each). (C) Mean fluorescence values of the expression data depicted in panel A. Data shown are the mean and standard deviation of a total of six samples, corresponding to three biological replicates with two technical replicates each. ${ }^{* \star}, P \leq 0.005$; n. s., not significant (one-way ANOVA with Bonferroni's multiple comparisons test).

independently of the RsbR1 paralogues and further suggested that the RsbR1 paralogues might attenuate the downstream signalling that leads to SigB activation.

Distinct interactions in the cytosol and membrane involving L. monocytogenes stressosome proteins. In contrast to current accepted models, the above data suggested that L. monocytogenes RsbR1 is mostly cytosolic and fully phosphorylated in actively growing cells not subjected to stress (Figs. 3, 4). We then sought to identify whether RsbR1 could have distinct interactions with RsbT in the cytosol and/or the membrane. RsbR1-RsbT interaction was examined by immunoprecipitation assays in cytosol and membrane fractions prepared from four different strains: wild type, $r s b R 1$-T175A, $r s b T$-N49A and $\Delta 4$. Using anti-RsbR1 antibodies coupled to magnetic beads, it was possible to co-immunoprecipitate RsbT from the cytosol but not the membrane of wild-type bacteria (Fig. 6A). The cytosolic RsbR1-RsbT interaction was detected in the rsbR1T175A mutant (Fig. 6B) but not in the kinase-inactive rsbT-N49A mutant (Fig. 6C). Interestingly, the lack of functional RsbR1 paralogues in the $\Delta 4$ mutant resulted in the detection of a stable RsbR1-RsbT interaction in the membrane extract that was not visualized in the corresponding extract of wild-type bacteria (Fig. 6A,D). This result opened the possibility of RsbR1 paralogues associated to the membrane and competing with RsbR1 
A

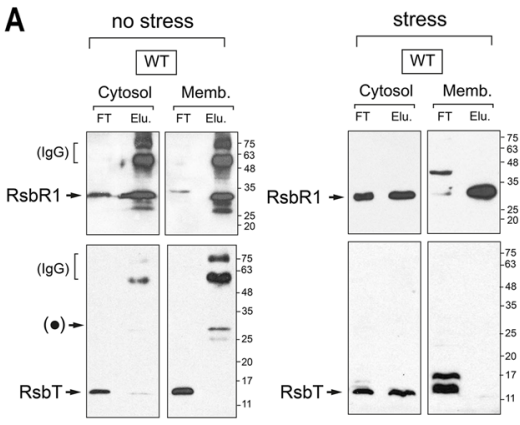

B

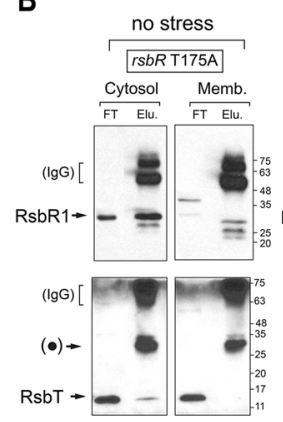

C

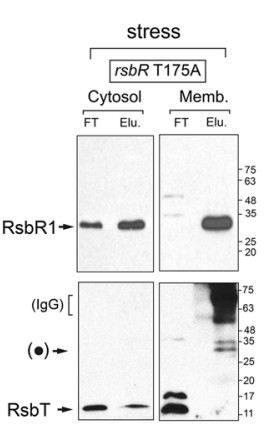

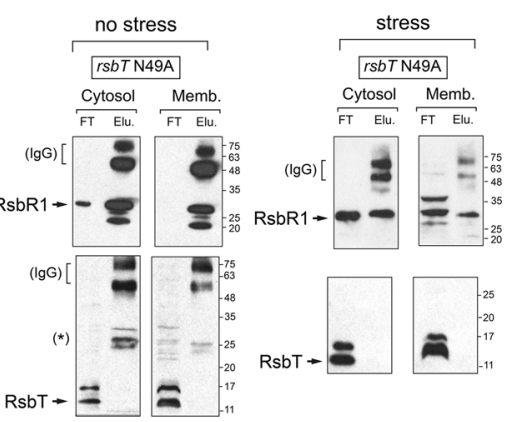

D

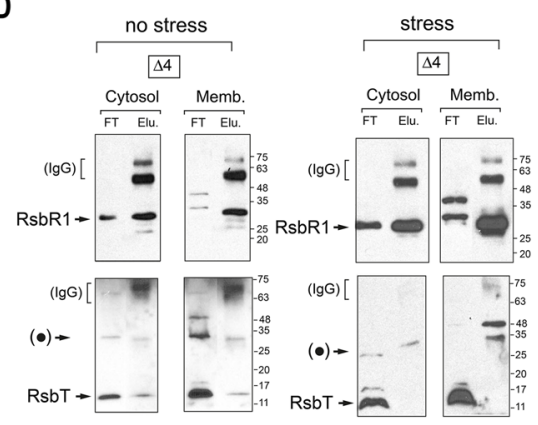

E

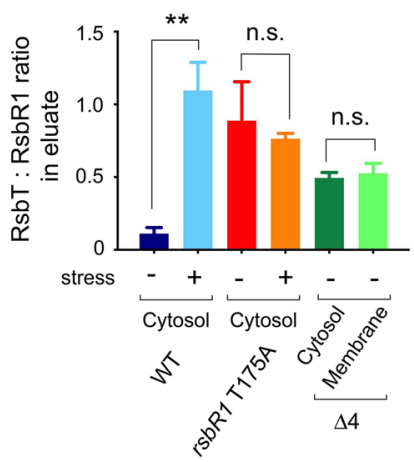

Figure 6. Analysis of L. monocytogenes RsbR1-RsbT interaction and action of RsbR1 paralogues in unstressed and stressed cells. Levels of interacting RsbR1 and RsbT detected in fractions of cytosol and membrane (25-fold concentrated versus cytosol) prepared from unstressed and stressed (30 min, $0.5 \mathrm{M} \mathrm{NaCl}$ ) cells after pull-down using anti-RsbR1 antibodies coupled to magnetic beads. Strains analysed: (A) wild-type; (B) rsbR1-T715A; (C) $r s b T$-N49A, and (D) the $\Delta 4$ mutant lacking functional RsbR1 paralogues. The blots shown correspond to a representative experiment of a total of three biological replicates. Bands indicated as $(\bullet)$ correspond to unspecific protein recognition. Molecular weight markers are indicated at the right side of each image. (E) Quantification of the RsbT:RsbR1 in eluate samples, which provides an estimation of the amount of RsbT:RsbR1 complex present in the sample. Note that RsbT signal in eluate was only evident in cytosol samples (unstressed, stressed) and in cytosol/membrane eluates of the $\Delta 4$ mutant. Data shown are mean and standard deviation of a total of three biological replicates. ${ }^{* *}, P<0.005$, n.s. $=$ not significant (one-way ANOVA with Tukey's multiple comparisons test). FT, flow-trough; Elu., eluate. See Supplementary Fig. S7 for images of full blots.

for the interaction with RsbT. This competition could modulate negatively the assembly of functional stressosome complexes and, consequently regulate the strength of the response.

The RsbR1-RsbT interaction was also analysed in bacteria responding to osmotic stress. In stressed cells, the amounts of RsbR1/RsbT complexes present in the cytosol increased significantly in wild-type bacteria (Fig. 6A,E). Notably, the exposure to stress led to loss of RsbR1-RsbT complexes in the cytosol and membrane fractions of the $\Delta 4$ mutant lacking functional paralogues (Fig. 6D). This result supported the idea of RsbR1 paralogues attenuating the stress response by probably reducing the number of active RsbR1-RsbS-RsbT stressosomes, from which $\mathrm{RsbT}$ would be rapidly released in the presence of the stress signal.

The idea of RsbR1 paralogues associated with the membrane was further tested by a proteomics approach in unstressed bacteria. We reasoned that in the absence of stress, in which a reduced but basal SigB activation is still detected (Fig. 2B), the competition of RsbR1 paralogues with RsbR1 for forming an active stressosome complex could be more evident at the membrane level. The immunoprecipitated material obtained using anti-RsbR1 antibodies from cytosol and membrane fractions of unstressed wild-type bacteria was processed for high resolution liquid chromatography-tandem mass spectrometry. The proteomic analysis, performed in three independent biological replicates, revealed the presence in the membrane of the RsbR1 paralogues RsbR2, RsbR3 and RsbL (Table 1). These results were in concordance with previous reports showing immunoprecipitation of these three RsbR1 paralogues, as well as RsbS, by pulling-down Prli42 ${ }^{15}$. Importantly, none of these RsbR1 paralogues were pulled down from the cytosol of wild type bacteria (Table 1) despite the much larger amount of RsbR1 detected in the cytosol by immunoblot assays (see Fig. 3A). These findings supported a stable interaction of RsbR1 with RsbS and the RsbR1 paralogues only in association to the membrane. Globally, these data allowed us to conclude the presence in actively growing L. monocytogenes cells of membrane-associated protein heterocomplexes in which RsbR1 paralogues could modulate the formation and/or stability of the RsbR1-RsbS-RsbT complex characterized in in vitro studies. 


\begin{tabular}{|c|c|c|c|c|c|c|c|c|c|c|c|}
\hline \multirow[b]{4}{*}{ Stressosome-related protein } & \multirow[b]{4}{*}{ Mass (Da) } & \multicolumn{6}{|c|}{ Wild type $^{\mathrm{a}}$} & \multicolumn{4}{|l|}{$\Delta r s b R 1^{\mathrm{b}}$} \\
\hline & & \multicolumn{3}{|l|}{ Cytosol } & \multicolumn{3}{|c|}{ Membrane } & \multicolumn{2}{|l|}{ Cytosol } & \multicolumn{2}{|c|}{ Membrane } \\
\hline & & \multicolumn{3}{|c|}{ Unique peptides (coverage, \%) } & \multicolumn{3}{|c|}{ Unique peptides (coverage, \%) } & \multicolumn{2}{|c|}{$\begin{array}{l}\text { Unique peptides } \\
\text { (coverage, \%) }\end{array}$} & \multicolumn{2}{|c|}{$\begin{array}{l}\text { Unique peptides } \\
\text { (coverage, \%) }\end{array}$} \\
\hline & & Exp. 1 & Exp. 2 & Exp. 3 & Exp. 1 & Exp. 2 & Exp. 3 & Exp. 1 & Exp. 2 & Exp. 1 & Exp. 2 \\
\hline RsbR1 (Lmo0889) & 31,620 & $19(74.0)$ & $19(74.0)$ & $27(78.0)$ & $13(55.0)$ & $11(44.0)$ & $25(74.0)$ & $2(10.0)$ & $1(4.3)$ & $1(4.0)$ & $1(4.3)$ \\
\hline RsbS (Lmo0890) & 12,596 & - & - & - & - & - & $3(20.0)$ & - & - & - & - \\
\hline RsbT (Lmo0891) & 14,727 & - & - & - & - & - & - & - & - & - & - \\
\hline RsbR2 (Lmo0161) & 31,737 & - & - & - & $1(2.9)$ & $2(8.3)$ & $12(49.2)$ & $1(5.1)$ & - & - & - \\
\hline RsbL (Lmo0799) & 28,816 & - & - & - & $6(33.0)$ & $3(16.0)$ & $11(56.1)$ & $1(3.6)$ & $1(5.5)$ & - & - \\
\hline RsbR3 (Lmo1642) & 30,414 & - & - & - & - & - & $11(50.2)$ & - & - & - & - \\
\hline RsbR4 (Lmo1842) & 31,516 & - & - & - & - & - & - & - & - & - & - \\
\hline
\end{tabular}

Table 1. L. monocytogenes stressosome proteins interacting with RsbR1 detected in cytosol and membrane fractions by high resolution MS. Complete lists of proteins identified in each of the three independent experiments performed are included in Supplementary information. - not detected. ${ }^{a}$ Examples of fragmentation spectra of high confidence for each of these proteins are shown in Supplementary Fig. S8. ${ }^{\text {b}}$ These peptides were detected with low quality (see example in Supplementary Fig. S9).

\section{Discussion}

In this study, we have examined in live L. monocytogenes cells the subcellular location and interaction dynamics of the core stressosome proteins RsbR1 and RsbS and the kinase RsbT. These three proteins have been extensively studied in vitro mainly in structural analyses in which purified proteins were combined and assembled into highly ordered macromolecular complexes ${ }^{15,16}$. To date, it is widely accepted that the RsbR1-RsbS-RsbT complex that adopts an icosahedral structure is the entity perceiving the external stress signal in live L. monocytogenes cells ${ }^{16,17}$. Integration of stress signals is thought to trigger phosphorylation at a second site (T209) in monophosphorylated RsbR1 (T175 P) and the single phosphorylatable residue present in RsbS (S56), events that could occur preferentially at the membrane level due to the tethering role assigned to the Prli42 miniprotein ${ }^{16}$. Our in vivo findings in live L. monocytogenes cells, however, differ somewhat from this view since we were unable to detect such stable RsbR1-RsbS-RsbT complex associated with the membrane. Instead, we identified a minimal membrane-associated RsbR1-RsbS complex interacting with at least three RsbR1 paralogues, RsbR2, RsbR3 and RsbL; and, in which RsbT was not detected (Table 1). The absence of RsbT from this membrane-associated complex is consistent with the high phosphorylation level detected for RsbR1 (Fig. 4A), the lack of RsbT in RsbR1immuno-precipitates prepared from membrane fractions (Fig. 6A) and, as reported by others ${ }^{15}$, the absence of RsbT in anti-Prli42 immuno-precipitates. Nonetheless, our immunoblot assays identified a RsbR1-RsbT interaction in the cytosol that was not reflected in the proteomic approach. RsbT is a small protein $(\sim 15 \mathrm{kDa})$, which limits the detection of tryptic peptides. It is also possible that only a small fraction of the immuno-precipitated cytosolic RsbR1 may establish a stable interaction with RsbT. This low abundance of interacting RsbT is another factor that might have limited its identification in membrane fractions by proteomic approaches.

On the other hand, the failure to detect a stable RsbR1-RsbT interaction in the L. monocytogenes membrane agrees with a previous report in B. subtilis based on immunoprecipitation assays using anti-RsbRA antibodies. This study, performed in total cell extracts, showed the predominance of RsbRA-RbsS complexes lacking RsbT ${ }^{35}$. Overall, our in vivo findings and those collected in B. subtilis support a short half-life for the RsbR1-RsbS-RsbT complex in live cells, in contrast to the stable complexes that are assembled in vitro with purified proteins. The transient nature of this stressosome could also explain why, in cells exposed to osmotic stress, the majority of complexes containing RsbR1 and RsbT are located in the cytosol (Fig. 6A). This finding opens the possibility of "cytosolic" stressosome complexes assembled with fully phosphorylated RsbR1/RsbS and stable RsbR1-RsbT interactions (Fig. 6A).

Moreover, our data showed that, in osmotic stress conditions and the absence of RsbR1 paralogues, the RsbR1RsbT interaction becomes undetectable probably due to the exacerbated release of RsbT and consistent with the slightly higher SigB activity displayed by the $\Delta 4$ mutant (Fig. 5). Based on these observations, we propose a model that allows for distinct stressosome configurations, all sharing the presence of fully phosphorylated RsbR1 and RsbS but with differences in other components such as RsbR1 paralogues, the kinase RsbT or, the association (or not) with a miniprotein (Fig. 7). This tentative model establishes a putative negative role of at least three of the four known RsbR1 paralogues in modulating the in vivo assembly of the membrane-associated stressosome but, based on our proteomic data, with probably no structural or regulatory role in the assembly of the hypothetical cytosolic stressosome containing phosphorylated RsbR1/RsbS. Except for RsbL, which is involved in the response to blue light ${ }^{26}$, no other functions were previously known for the other RsbR1 paralogues, RsbR2 and RsbR3 $3^{26,36-39}$.

Our study also provides insights into the distribution of stressosome proteins in subcellular compartments. The vast majority of RsbR1 and RsbS was detected in the cytosol and no changes in these protein pools were observed under stress. This may indicate that, in the nutrient-rich medium used in laboratory conditions, L. monocytogenes produces an excess of stressosome proteins compared to what it is needed to cope with at least the type of stress tested, hyperosmolarity. It would be of interest in the future to monitor whether a proportion of these cytosolic pools mobilize to the membrane in response to other stresses or during the interaction of 
A

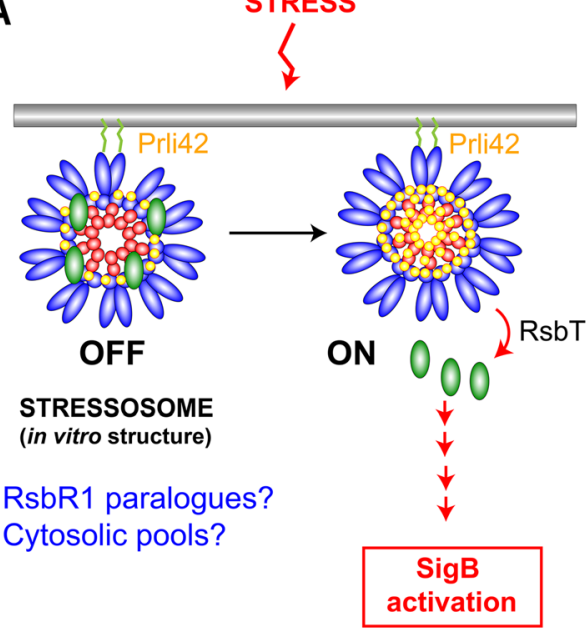

B

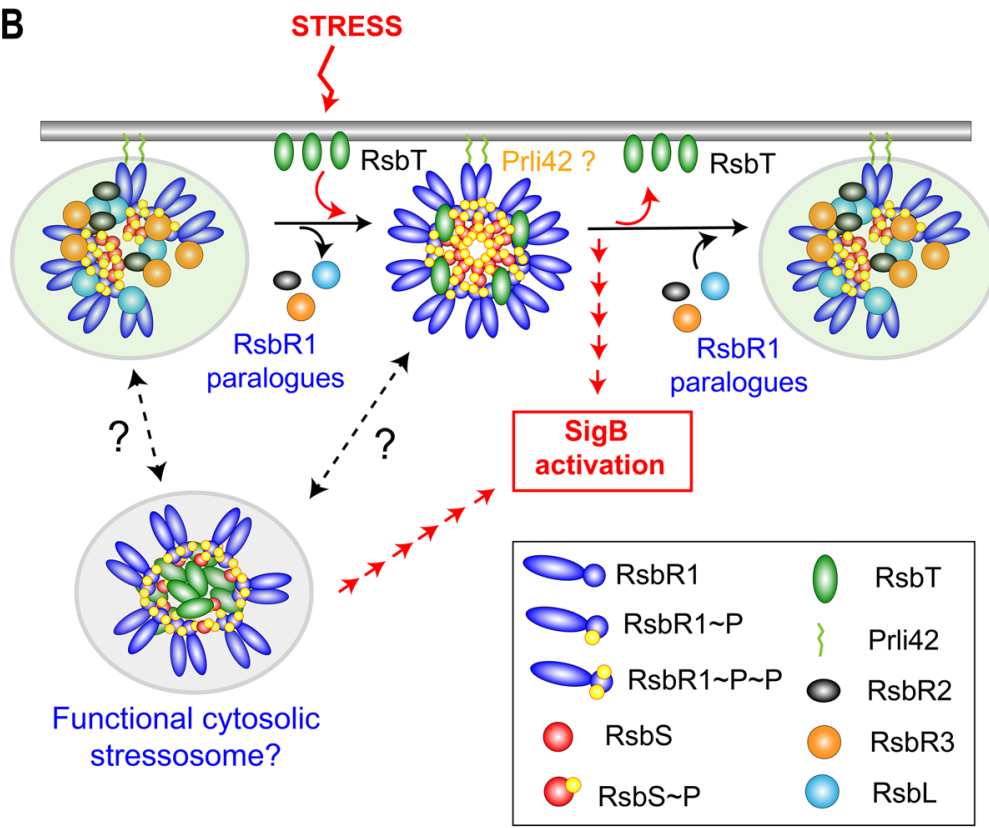

Figure 7. Tentative model depicting the dynamics of interaction among the stressosome proteins analysed in this study. (A) Model proposed for stress response based on crystallographic data obtained in vitro with purified proteins in L. monocytogenes ${ }^{16}$; (B) Model integrating the observations obtained in vivo from L. monocytogenes about the distribution and interaction of distinct stressosome proteins. Note the putative negative role assigned to RsbR1 paralogues to prevent the formation of a stable RsbR1-RsbS-RsbT complex, which could be only transiently formed upon stress. The association of RsbT with the membrane is depicted as a direct interaction, although it could be indirect since it lacks defined hydrophobic domains. The model also highlights the presence of a large pools of cytosolic RsbR1, RsbS and RsbT, with the possibility of functional stressosomes in this location capable of activating SigB. See text for details.

L. monocytogenes with the mammalian host or, as above postulated, contribute to the assembly of functional stressosomes in this subcellular location. These are questions that we aim to address in future work.

One of the most puzzling observations of our study was the predominance of highly phosphorylated forms of RsbR1 and RsbS, even in actively growing L. monocytogenes not subjected to osmotic stress. Moreover, the assays performed with control mutant strains harbouring a mutation in the first phosphorylatable site of RsbR1 (T175) or a kinase-inactive RsbT variant, demonstrated that most RsbR1 molecules are doubly phosphorylated and that RsbS is also fully phosphorylated, irrespective of exposure to osmotic stress. Importantly, although we observed that RsbT-mediated phosphorylation is absolutely required for SigB activation by osmotic stress, our findings in live L. monocytogenes contradict the view of phosphorylation events occurring on RsbR 1 and RsbS concomitantly to stress perception. Interestingly, the kinase-inactive RsbT-N49A variant loses the capacity to interact with RsbR1 regardless of stress and is unable to activate SigB (Figs. 5, 6). So, the release of RsbT from the stressosome seems not to be per se sufficient to activate SigB. As described for RsbT in Vibrio brasiliensis ${ }^{40}$, the possibility that L. monocytogenes RsbT has autophosphorylation activity cannot be discarded, which could be a requisite to activate RsbU and, as a result, the SigB cascade. Future studies should certainly test this tempting hypothesis.

It is also relevant to note that phosphorylation at the T175 residue of L. monocytogenes RsbR 1 is not absolutely required to respond to stress, to interact with RsbT in the cytosol (Fig. 6B), or to become phosphorylated at the second phosphorylatable site, the T209 residue (Fig. 4A). Nonetheless, the lack of RsbR1 phosphorylation at T175 decreases the intensity of the SigB response, suggesting that this phosphorylation might stabilize at a larger extent the functional RsbR1-RsbS stressosome once RsbT is released. It is important to highlight that, unlike what we observe in L. monocytogenes, lack of phosphorylation at the first phosphorylatable residue of $B$. subtilis RsbRA (T171) impairs the stress response ${ }^{18}$ and that a recent study claimed phosphorylation of T175 as relevant for L. monocytogenes to cope with acid stress ${ }^{33}$. Interestingly, the loss of RsbT kinase activity in L. monocytogenes renders RsbR1 less stable in no stress and stress conditions (Supplementary Fig. S5). This lower amount of RsbR1 can also affect the capacity of the cell to activate SigB.

An additional difference observed between L. monocytogenes RsbR1 and B. subtilis RsbRA was the phosphorylation state in basal conditions. Most of the former existed as doubly-phosphorylated isoform whereas the second most common one was the mono-phosphorylated. We are still uncertain about the basis of this difference. However, the fact that we did not detect a predominance of doubly-phosphorylated RsbRA in B. subtilis, even in stress conditions, allowed us to discard potential artefacts during sample processing linked to unspecific increased phosphorylation. In this context, it is noteworthy that the study of Eymann et al. in $B$. subtilis concluded that phosphorylation of the RsbRA T205 residue could contribute as a feedback mechanism to limit SigB activation, 
together with the phosphatase activity of $\mathrm{RsbX}^{22}$. As an alternative interpretation, the possibility that the stressosome could be tuned down in L. monocytogenes in basal conditions by an excess of RsbR1 phosphorylated at T209 should therefore not be discarded. Nonetheless, T209 (T205 in B. subtilis) phosphorylation has been proposed to be linked to extreme stress in order to limit SigB signalling ${ }^{16,22}$, a condition that we think is unlikely to be present in actively growing L. monocytogenes cells.

As we propose in the model depicted in Fig. 7, stimulation of the downstream signalling cascade that activates L. monocytogenes SigB might be modulated by cytosolic and membrane-associated complexes responding to distinct environmental stimuli. The dynamics of assembly and disassembly of these stressosome complexes could be fine-tuned by RsbR1 paralogues, especially at the membrane, making these paralogues a new regulatory element controlling stressosome function.

\section{Methods}

Bacterial strains, plasmids and primers. Listeria monocytogenes EGD-e, E. coli and B. subtilis BG214 strains and plasmids and the primers used for this study are listed in supplementary information, Tables S1 and $\mathrm{S} 2$, respectively.

Construction of genetically modified L. monocytogenes. The genes $r s b R 1$ and $r s b T$ were mutated to incorporate Thr-to-Ala in the codon 175 and Asn-to-Ala in the codon 49, respectively. The codon 175 ACA (Thr) in $r s b R 1$ was changed to GCG (Ala) and the codon 49 AAT (Asn) in $r s b T$ was changed to GCT (Ala). In both cases silent mutations were added the two adjunct codons in order to discern mutant from WT codons by PCR during mutagenesis ( $r s b 1 \mathrm{WT}$-ATTGACACAGAAAGA- sequence mutated to $r s b R 1$ T175A -ATA GATGCGGAGCGT- and $r s b$ WT -GCTAGAAATATTTTC- to $r s b T$ N49A -GCACGTGCTATCTTT-). The mutagenic sequences, each with a total length of $612 \mathrm{bp}$, including EcoRI and BamHI restriction sites at each end, were artificially synthetized in the vectors pEX-A128::rsbR1 (T175A) and pEX-K168::rsbT (N49A) (Eurofins Genomics). The mutagenic sequences were subsequently cloned into shuttle vector pMAD, originating pMAD::rsbR1 (T175A) and pMAD::rsbT (N49A). L. monocytogenes electrocompetent cells were created as previously described ${ }^{41}$. The electrocompetent $L$. monocytogenes WT was separately transformed with pMAD::rsbR1 (T175A) and pMAD::rsbT (N49A). The L. monocytogenes rsbL (C56A) mutant strain was transformed with pMAD:: $\Delta l m o 1842$. Electroporated cells were plated in BHI supplemented with erythromycin (Ery). Chromosomal integration and subsequent excision were achieved through a two-step recombination as previously described $^{38}$. cPCR with the primers $r s b R 1$ _upflank_F paired with either $r s b R 1$ (T175A)_R and $r s b T$ (N49A)_R were used to identify the chromosomal mutation in $r s b R 1$ and $r s b T$, respectively, while the deletion of $l m o 1842$ was verified with primers lmo1842_flank_F and lmo1842_flank_R. To construct the L. monocytogenes EGD-e quadruple-mutant (rsbL (C56A); $\Delta$ lmo1842; $\Delta$ lmo1642; $\Delta l m o 0161)$, the double mutant L. monocytogenes EGD-e ( $r s b L$ (C56A); $\Delta l m o 1842)$ was sequentially mutated using the mutagenic vectors pMAD:: $\Delta l m o 1642$ and pMAD:: $\Delta l m o 0161$. The primers pair lmo1642_flank_F; lmo1642_flank_R and lmo0161_flank_F; lmo0161_ flank_R, were used to verify the chromosomal deletion of each respective gene. The SigB-eGFP reporter vector pKSV7- $\mathrm{P}_{\text {lmo2230 }}:$ egfp was transformed in each mutant strain as previously described ${ }^{29}$. The chromosomal integration of the SigB-eGFP reporter occurred upstream of the original $l m o 2230$ promoter region by homologous recombination resulting in a duplication of the promoter region. Integration was verified by PCR (using primers eGFP-lmo2230-F integration and eGFP-lmo2230-R integration). All genomic DNA from each mutant strain were whole genome-sequenced to confirm the mutation and identify secondary mutations.

Hyperosmotic shock growth conditions. Listeria monocytogenes EGD-e and B. subtilis BG214 strains were cultured in brain heart infusion, BHI (Becton Dickinson, BD) and Luria-Bertani, LB, broth, respectively, with continuous shaking at $37^{\circ} \mathrm{C}$ until reaching mid-exponential phase $\left(\mathrm{OD}_{600 \mathrm{~nm}}=0.4\right)$ (no stress). Salt $(0.5 \mathrm{M}$ $\mathrm{NaCl}$ in the form of solid salt crystals) was added to the BHI medium for 30 min (osmotic stress). Around $3 \times 10^{10}$ bacteria $\left(50 \mathrm{~mL}\right.$ culture) were spun down by centrifugation $\left(10,000 \times g, 18 \mathrm{~min}, 4^{\circ} \mathrm{C}\right)$ and washed twice in phosphate buffer saline (PBS), $\mathrm{pH}$ 7.4. The pellet was kept at $-80^{\circ} \mathrm{C}$ until being subjected to subcellular fractionation.

The same method was performed in experiments involving addition of $100 \mu \mathrm{g} / \mathrm{mL}$ chloramphenicol to inhibit protein synthesis in no stress and hyperosmotic conditions.

Subcellular fractionation and Western blot analysis of stressosome machinery-related proteins in L. monocytogenes. Fractions containing cytosolic and membrane proteins from exponential phase growing bacteria were obtained as described previously ${ }^{42}$. The pellet of bacteria was washed in $10 \mathrm{~mL}$ of TS buffer $\left(10 \mathrm{mM}\right.$ Tris $\mathrm{HCl} \mathrm{pH} 6.9,10 \mathrm{mM} \mathrm{MgCl}_{2}, 0.5 \mathrm{M}$ sucrose) and centrifuged at $10,000 \times g, 18 \mathrm{~min}, 4^{\circ} \mathrm{C}$. Then, the pellet was resuspended in a lysis solution: TS buffer containing $60 \mu \mathrm{g} / \mathrm{mL}$ mutanolysin from Streptomyces globisporus (ATCC 21553, Sigma-Aldrich), $250 \mu \mathrm{g} / \mathrm{mL}$ RNAse A, protease inhibitor cocktail and incubated during $5 \mathrm{~h}$ at $37^{\circ} \mathrm{C}$ with slow-rotation agitation. Protoplasts were recovered by centrifugation at $15,000 \times g, 10 \mathrm{~min}, 4^{\circ} \mathrm{C}$. The supernatant corresponding to the cell wall fraction was discarded and the pellet containing the protoplasts was washed with $1 \mathrm{~mL}$ of PBS, pH 7.4 and centrifuged at $10,000 \times \mathrm{g}, 18 \mathrm{~min}, 4^{\circ} \mathrm{C}$. The protoplasts were resuspended into $400 \mu \mathrm{L}$ of PBS, pH 7.4, $1 \mu \mathrm{g} / \mathrm{mL}$ DNAse A, protease inhibitor cocktail and they were lysed by low power sonication. Unbroken cells were removed by centrifugation at $20,000 \times g, 10 \mathrm{~min}, 4^{\circ} \mathrm{C}$ and the supernatant was subjected to ultracentrifugation at $100,000 \times g, 1 \mathrm{~h}, 4^{\circ} \mathrm{C}$ to separate cytosol and membrane fractions. The pellet containing membrane proteins was washed with PBS, pH 7.4 by centrifugation at $100,000 \times g, 1 \mathrm{~h}, 4^{\circ} \mathrm{C}$. The pellet was resuspended into $400 \mu \mathrm{L}$ of PBS, pH 7.4 to get a membrane extract containing the same number of bacteria as in the cytosolic fraction. To obtain tenfold $(10 \times)$ concentrated membrane extracts, subcellular 
fractionation was performed from twice as many L. monocytogenes cells and the pellet containing membrane proteins was resuspended into $80 \mu \mathrm{L}$ of $\mathrm{PBS}, \mathrm{pH}$ 7.4. A volume of $10 \mu \mathrm{L}$ of cytosolic and membrane fractions was analyzed by SDS-PAGE and Western-blot using affinity purified polyclonal rabbit antibodies against stressosome machinery-related proteins (RsbR1, RsbT, RsbS) and SigB. Protein levels detected by western blot from three independent experiments were quantified with ImageJ and statistical analysis was undertaken by one-way ANOVA (Tukey's multiple comparison test).

Identification of phosphorylated isoforms of RsbR1 and RsbS with Phos-Tag system based on phosphate- $\mathrm{Zn}^{2+}$ ion sequestration. Listeria monocytogenes and B. subtilis cultures were boiled for $20 \mathrm{~s}$ at $100{ }^{\circ} \mathrm{C}$ to stop the metabolism before harvesting the cells by centrifugation. The cells were washed twice with a killing buffer containing $20 \mathrm{mM}$ Tris pH 7.5, $5 \mathrm{mM} \mathrm{MgCl}_{2}, 20 \mathrm{mM} \mathrm{NaN}_{3}\left(10,000 \times g, 18 \mathrm{~min}, 4^{\circ} \mathrm{C}\right)^{22}$. The pellet was subjected to subcellular fractionation as previously described. An additional control of sample preparation consisted in fixing L. monocytogenes cells with $4 \%$ (w/v) paraformaldehyde before subcellular fractionation.

Phos-tag electrophoresis based on phosphate- $\mathrm{Zn}^{2+}$ ion sequestration (SuperSep Phos-tag precast gels) was used to separate the phosphorylated isoforms of RsbR1 and RsbS. The Phos-tag polyacrylamide gel contains zinc ions that selectively bind to the phosphate ions and the complex is stable at neutral $\mathrm{pH}$. The migration speed of phosphorylated proteins decreases due to the binding of the metallic ion and phosphorylated/nonphosphorylated proteins are separated as different bands.

Purification of His-tagged RsbR1 protein by immobilized metal affinity purification (IMAC). His-tagged RsbR1 was expressed in E. coli BL21 (DE3) Gold competent cells (Novagen). Bacteria were grown overnight at $16^{\circ} \mathrm{C}$ in $\mathrm{LB}$ broth $(100 \mathrm{~mL})$ and protein expression was induced during $4 \mathrm{~h}$ with $1 \mathrm{mM}$ IPTG after reaching an $\mathrm{OD}_{600}=0.2$. Bacteria were then spun down $\left(10,000 \times g, 18 \mathrm{~min}, 4^{\circ} \mathrm{C}\right)$ and washed with PBS, pH 7.4. Cells were resuspended in $4 \mathrm{~mL}$ of binding buffer [ $50 \mathrm{mM} \mathrm{NaH}_{2} \mathrm{PO}_{4}, 200 \mathrm{mM} \mathrm{NaCl}, 5 \mathrm{mM}$ imidazole, $3 \mu \mathrm{g} / \mathrm{mL}$ DNAse A, $100 \mathrm{mM}$ Phenylmethylsulfonylfluoride (PMSF), $\mathrm{pH} 7.0$ ] and lysed by sonication. The bacterial lysate was separated from cell debris by high-speed centrifugation $\left(13,000 \times g, 30 \mathrm{~min}, 4^{\circ} \mathrm{C}\right)$.

Immobilized metal affinity chromatography (IMAC) resin (TALON) was pre-equilibrated by three washes with binding buffer $(700 \times \mathrm{g}, 2 \mathrm{~min}, \mathrm{RT})$. The bacterial lysate was incubated with the resin by slow-rotation agitation during $4 \mathrm{~h}$ at $4{ }^{\circ} \mathrm{C}$ to allow binding of the His-tagged protein to the resin. Incubation was done at $4{ }^{\circ} \mathrm{C}$ to prevent proteolysis. The flow-through was collected by centrifugation $\left(700 \times g, 5 \mathrm{~min}, 4^{\circ} \mathrm{C}\right)$ and the resin was washed three times with $10 \mathrm{~mL}$ of binding buffer $\left(700 \times \mathrm{g}, 5 \mathrm{~min}, 4^{\circ} \mathrm{C}\right)$. The purified His-tagged protein was eluted with an imidazole gradient (from 50 to $500 \mathrm{mM}$ ). The fractions were analyzed by Coomassie and those containing the purified His-tagged protein (eluted from 150 to $250 \mathrm{mM}$ imidazole) were concentrated to reach a final volume of $500 \mu \mathrm{L}$ (Millipore Amicon).

The concentrated eluate fraction was dialyzed overnight against $1 \mathrm{~L}$ of coupling buffer $(100 \mathrm{mM}$ MOPS buffer, $\mathrm{pH} 7.5$ ) at $4{ }^{\circ} \mathrm{C}$ to remove imidazole and incubated with derivatized cross-linked agarose gel bead support (Affigel 10 , BioRad) at $4{ }^{\circ} \mathrm{C}$ for $4 \mathrm{~h}$ with slow-rotation agitation. The purified protein RsbR1 coupling to the resin was cross-linked by adding $100 \mu \mathrm{L}$ of quenching buffer $(1 \mathrm{M}$ ethanolamine $\mathrm{pH} 8.0)$ at $4^{\circ} \mathrm{C}$ during $1 \mathrm{~h}$. The coupling mixture was washed with $10 \mathrm{~mL}$ of binding buffer $\left(700 \times \mathrm{g}, 5 \mathrm{~min}, 4^{\circ} \mathrm{C}\right)$ and transferred to a column (TALON, $2 \mathrm{~mL}$ gravity columns). The column was washed with PBS, pH 7.4 and was stored at $4^{\circ} \mathrm{C}$ in $2 \mathrm{~mL}$ of $\mathrm{PBS} \mathrm{pH} 7.4$, $0.2 \%(\mathrm{w} / \mathrm{v})$ sodium azide.

Antibody purification by antigen-specific affinity. Polyclonal rabbit crude serum anti-RsbR1 (1.5 mL) (Charles River, France) was inactivated at $56^{\circ} \mathrm{C}$ for $30 \mathrm{~min}$ to destroy complement factors. The serum was spun down $\left(13,000 \times g, 10 \mathrm{~min}, 4^{\circ} \mathrm{C}\right)$ and the supernatant was applied three times at $4{ }^{\circ} \mathrm{C}$ to the column coupled to purified His-tagged RsbR1 protein. A gauge needle was fitted to slow rate at which the column flows. The column was washed with $10 \mathrm{~mL}$ of PBS, pH 7.4 and affinity purified antibodies were eluted with $10 \mathrm{~mL}$ of elution buffer (100 mM glycine, $\mathrm{pH} 2.4$ ). The $\mathrm{pH}$ of the elution fractions was immediately neutralized with $1 \mathrm{M}$ Tris $\mathrm{pH} 8.0$ and the fractions concentrated to reach a final volume of $500 \mu \mathrm{L}$ corresponding to a concentration of antibodies of $\sim 1 \mathrm{mg} / \mathrm{mL}$. The purified anti-RsbR1 antibody was stored at $-20{ }^{\circ} \mathrm{C}$ in $20 \%$ glycerol.

Purification of the stressosome from cell extracts by pull-down experiments. Wild-type, $r s b R 1$ T175A, $r s b T-\mathrm{N} 49 \mathrm{~A}, \Delta 4$ and $\Delta r s b R 1$ strains were grown to exponential phase $\left(\mathrm{OD}_{600 \mathrm{~nm}}=0.4\right)$ in $300 \mathrm{~mL}$ of BHI medium $\left(1.8 \times 10^{11}\right.$ bacteria). The cells were washed three times with PBS, pH 7.4 by centrifugation at $10,000 \times g$, $18 \mathrm{~min}, 4^{\circ} \mathrm{C}$. Bacteria were disrupted using a French press (three passes) in $10 \mathrm{~mL}$ of a buffer containing $50 \mathrm{mM}$ Tris $\mathrm{HCl}, \mathrm{pH} 7.0,60 \mu \mathrm{g} / \mathrm{mL}$ DNAse A, $2 \mathrm{mM}$ PMSF. Cell debris were removed by centrifugation at $5000 \times g$, $5 \mathrm{~min}, 4^{\circ} \mathrm{C}$ and the supernatant was subjected to ultracentrifugation at $145,000 \times g, 1 \mathrm{~h}, 4^{\circ} \mathrm{C}$. The supernatant constituting the cytosolic fraction $(10 \mathrm{~mL})$ was kept at $-20^{\circ} \mathrm{C}$ and the pellet containing the membrane proteins was incubated with $400 \mu \mathrm{L}$ of PBS, $\mathrm{pH} 7.4,0.1 \%$ Triton $\mathrm{X}-100$ during $30 \mathrm{~min}$ at $4{ }^{\circ} \mathrm{C}$ with slow-rotation agitation. The sample was subjected to ultracentrifugation at $100,000 \times g, 1 \mathrm{~h}, 4^{\circ} \mathrm{C}$ and supernatant containing membrane proteins and complexes $(400 \mu \mathrm{L})$ was kept at $-20^{\circ} \mathrm{C}(25 \times$ concentrated membrane extract compared to the cytosolic fraction).

To immuno-precipitate the stressosome complex, $1.5 \mathrm{mg}(50 \mu \mathrm{L})$ of Dynabeads protein $\mathrm{G}$ (Invitrogen) were incubated with $120 \mu \mathrm{g}$ of affinity purified rabbit polyclonal anti-RsbR1 antibodies during $15 \mathrm{~min}$ at room temperature with slow-rotation agitation. Then, the antibodies were crosslinked to the magnetic beads with $3 \mathrm{mM}$ $\mathrm{BS}^{3}$ [bis(sulfosuccinimidyl)suberate] (Thermo Fisher) during $30 \mathrm{~min}$ at room temperature. The tube was placed on the magnet to remove the supernatant containing unbound antibodies and crosslinker, and the beads were washed three times with the washing buffer: PBS, pH 7.4, with 0.02\% (v/v) Tween 20. 
The cytosolic $(10 \mathrm{~mL})$ and the 25 -fold $(25 \times)$ concentrated membrane $(400 \mu \mathrm{L})$ fractions were incubated separately with the beads at $4{ }^{\circ} \mathrm{C}$ during $5 \mathrm{~h}$ with slow-rotation agitation. The supernatant constituting the flowthrough was kept for further analysis and the beads were washed three times with the washing buffer. Finally, the beads were incubated with $40 \mu \mathrm{L}$ of elution buffer $(100 \mathrm{mM}$ glycine, $150 \mathrm{mM} \mathrm{NaCl}, \mathrm{pH} 2.4)$ during $5 \mathrm{~min}$. The supernatant constituting the eluate was transferred to a clean tube and the $\mathrm{pH}$ was adjusted by adding $10 \mu \mathrm{L}$ of $1 \mathrm{M}$ Tris, $\mathrm{pH} 7.5$ to preserve the integrity of the proteins. The pull-down procedure resulted in the obtention of eluates from the cytosolic and membrane extracts $250 \times$ and $10 \times$ concentrated, respectively, compared to their respective flow-through sample. The eluate and flow through samples from the cytosolic and membrane fractions were analyzed by SDS-PAGE and Western blot using affinity purified polyclonal rabbit anti-RsbR1 and anti-RsbT antibodies.

The eluates were also analyzed by Liquid Chromatography-Tandem Mass Spectrometry (LC-MS/MS). The samples were cleaned and concentrated using a concentrator SDS-PAGE gel and one third of each individual lane was in-gel digested using trypsin and reducing conditions. Resulted peptides were dried in a speed vacuum and analyzed by LC-MS, using an Eksigent 1D Plus nanochromatographer and a Sciex 5600 TripleTOF mass spectrophotometer. The gradient time used for peptide identification was $100 \mathrm{~min}$. MS and MS/MS spectra were used to launch a database search using Mascot against the UNIPROT L. monocytogenes EGD-e database (entry NC 003210.1).

Flow cytometry analysis of eGFP-expressing cells. Bacterial strains were grown to $\mathrm{OD}_{600 \mathrm{~nm}}=0.4$ (no stress) in BHI or BHI supplemented with $0.5 \mathrm{M} \mathrm{NaCl}$ during $30 \mathrm{~min}$ (stress). Around $3 \times 10^{8}$ bacteria were spun down by centrifugation $\left(10,000 \times \mathrm{g}, 18 \mathrm{~min}, 4^{\circ} \mathrm{C}\right)$ and washed twice in PBS, pH 7.4. The cells were fixed with $4 \%(\mathrm{w} / \mathrm{v})$ paraformaldehyde for $15 \mathrm{~min}$ at room temperature. Fixed cells were harvested by centrifugation and resuspended in $500 \mu \mathrm{L}$ of filtered PBS, pH 7.4. Quantification of single cell fluorescence was achieved by flow cytometry with Beckman Coulter GALLIOS Analyzer with $488 \mathrm{~nm}$ blue laser excitation and 50,000 events recorded for each sample. The data collected were processed with Kaluza software to plot side and forward scatter values, the percentage of eGFP-positive cells and the mean of fluorescence values. Statistical analysis has been carried out by one-way ANOVA (Bonferroni's multiple comparison test).

Statistical analyses and densitometry. Statistical significance was analyzed with GraphPad Prism v8.4.3 software (GraphPad Inc.) using one-way analysis of variance (ANOVA) with Tukey's or Bonferroni's multiple comparison tests. A $P$ value $\leq 0.05$ was considered significant. Densitometry on bands obtained in immunoblot assays was performed using ImageJ, available from the National Institute of Health, USA.

Received: 30 April 2020; Accepted: 17 November 2020

Published online: 30 November 2020

\section{References}

1. Hecker, M. \& Volker, U. General stress response of Bacillus subtilis and other bacteria. Adv. Microb. Physiol. 44, 35-91. https://doi. org/10.1016/s0065-2911(01)44011-2(2001).

2. Hecker, M., Pane-Farre, J. \& Volker, U. SigB-dependent general stress response in Bacillus subtilis and related gram-positive bacteria. Annu. Rev. Microbiol. 61, 215-236. https://doi.org/10.1146/annurev.micro.61.080706.093445 (2007).

3. Boylan, S. A., Redfield, A. R., Brody, M. S. \& Price, C. W. Stress-induced activation of the sigma B transcription factor of Bacillus subtilis. J. Bacteriol. 175, 7931-7937. https://doi.org/10.1128/jb.175.24.7931-7937.1993 (1993).

4. Gaballa, A., Guariglia-Oropeza, V., Wiedmann, M. \& Boor, K. J. Cross talk between SigB and PrfA in listeria monocytogenes facilitates transitions between extra- and intracellular environments. Microbiol. Mol. Biol. Rev. https://doi.org/10.1128/MMBR.00034 -19 (2019).

5. Liu, Y. et al. Systematic review of the Listeria monocytogenes sigma(B) regulon supports a role in stress response, virulence and metabolism. Future Microbiol. 14, 801-828. https://doi.org/10.2217/fmb-2019-0072 (2019).

6. Benson, A. K. \& Haldenwang, W. G. Bacillus subtilis sigma B is regulated by a binding protein (RsbW) that blocks its association with core RNA polymerase. Proc. Natl. Acad. Sci. USA 90, 2330-2334. https://doi.org/10.1073/pnas.90.6.2330 (1993).

7. Dufour, A. \& Haldenwang, W. G. Interactions between a Bacillus subtilis anti-sigma factor (RsbW) and its antagonist (RsbV). J. Bacteriol. 176, 1813-1820. https://doi.org/10.1128/jb.176.7.1813-1820.1994 (1994).

8. Yang, X., Kang, C. M., Brody, M. S. \& Price, C. W. Opposing pairs of serine protein kinases and phosphatases transmit signals of environmental stress to activate a bacterial transcription factor. Genes Dev. 10, 2265-2275. https://doi.org/10.1101/gad.10.18.2265 (1996).

9. Voelker, U. et al. Separate mechanisms activate sigma B of Bacillus subtilis in response to environmental and metabolic stresses. J. Bacteriol. 177, 3771-3780. https://doi.org/10.1128/jb.177.13.3771-3780.1995 (1995).

10. Voelker, U., Dufour, A. \& Haldenwang, W. G. The Bacillus subtilis rsbU gene product is necessary for RsbX-dependent regulation of sigma B. J. Bacteriol. 177, 114-122. https://doi.org/10.1128/jb.177.1.114-122.1995 (1995).

11. Vijay, K., Brody, M. S., Fredlund, E. \& Price, C. W. A PP2C phosphatase containing a PAS domain is required to convey signals of energy stress to the sigmaB transcription factor of Bacillus subtilis. Mol. Microbiol. 35, 180-188. https://doi.org/10.104 6/j.1365-2958.2000.01697.x (2000).

12. Chaturongakul, S. \& Boor, K. J. SigmaB activation under environmental and energy stress conditions in Listeria monocytogenes. Appl. Environ. Microbiol. 72, 5197-5203. https://doi.org/10.1128/AEM.03058-05 (2006).

13. Marles-Wright, J. et al. Molecular architecture of the "stressosome," a signal integration and transduction hub. Science 322, 92-96. https://doi.org/10.1126/science.1159572 (2008).

14. Marles-Wright, J. \& Lewis, R. J. The stressosome: molecular architecture of a signalling hub. Biochem. Soc. Trans. 38, 928-933. https://doi.org/10.1042/BST0380928 (2010).

15. Impens, F. et al. N-terminomics identifies Prli42 as a membrane miniprotein conserved in Firmicutes and critical for stressosome activation in Listeria monocytogenes. Nat. Microbiol. 2, 17005. https://doi.org/10.1038/nmicrobiol.2017.5 (2017). 
16. Williams, A. H. et al. The cryo-electron microscopy supramolecular structure of the bacterial stressosome unveils its mechanism of activation. Nat. Commun. 10, 3005. https://doi.org/10.1038/s41467-019-10782-0 (2019).

17. Pane-Farre, J., Quin, M. B., Lewis, R. J. \& Marles-Wright, J. Structure and function of the stressosome signalling hub. Subcell Biochem. 83, 1-41. https://doi.org/10.1007/978-3-319-46503-6_1 (2017).

18. Kim, T. J., Gaidenko, T. A. \& Price, C. W. In vivo phosphorylation of partner switching regulators correlates with stress transmission in the environmental signaling pathway of Bacillus subtilis. J. Bacteriol. 186, 6124-6132. https://doi.org/10.1128/JB.186.18.61246132.2004 (2004).

19. Akbar, S., Kang, C. M., Gaidenko, T. A. \& Price, C. W. Modulator protein RsbR regulates environmental signalling in the general stress pathway of Bacillus subtilis. Mol. Microbiol. 24, 567-578. https://doi.org/10.1046/j.1365-2958.1997.3631732.x (1997).

20. Gaidenko, T. A., Yang, X., Lee, Y. M. \& Price, C. W. Threonine phosphorylation of modulator protein RsbR governs its ability to regulate a serine kinase in the environmental stress signaling pathway of Bacillus subtilis. J. Mol. Biol. 288, 29-39. https://doi. org/10.1006/jmbi.1999.2665 (1999).

21. Chen, C. C., Yudkin, M. D. \& Delumeau, O. Phosphorylation and RsbX-dependent dephosphorylation of RsbR in the RsbR-RsbS complex of Bacillus subtilis. J. Bacteriol. 186, 6830-6836. https://doi.org/10.1128/JB.186.20.6830-6836.2004 (2004).

22. Eymann, C. et al. In vivo phosphorylation patterns of key stressosome proteins define a second feedback loop that limits activation of Bacillus subtilis sigmaB. Mol. Microbiol. 80, 798-810. https://doi.org/10.1111/j.1365-2958.2011.07609.x (2011).

23. Delumeau, O. et al. Functional and structural characterization of RsbU, a stress signaling protein phosphatase 2C. J. Biol. Chem. 279, 40927-40937. https://doi.org/10.1074/jbc.M405464200 (2004).

24. O’Byrne, C. P. \& Karatzas, K. A. The role of sigma B (sigma B) in the stress adaptations of Listeria monocytogenes: overlaps between stress adaptation and virulence. Adv. Appl. Microbiol. 65, 115-140. https://doi.org/10.1016/S0065-2164(08)00605-9 (2008).

25. Woodbury, R. L., Luo, T., Grant, L. \& Haldenwang, W. G. Mutational analysis of RsbT, an activator of the Bacillus subtilis stress response transcription factor, sigmaB. J. Bacteriol. 186, 2789-2797. https://doi.org/10.1128/jb.186.9.2789-2797.2004 (2004).

26. Dorey, A. L., Lee, B. H., Rotter, B. \& O’Byrne, C. P. Blue light sensing in Listeria monocytogenes is temperature-dependent and the transcriptional response to it is predominantly SigB-dependent. Front. Microbiol. 10, 2497. https://doi.org/10.3389/fmicb .2019.02497 (2019).

27. Utratna, M., Shaw, I., Starr, E. \& O'Byrne, C. P. Rapid, transient, and proportional activation of sigma(B) in response to osmotic stress in Listeria monocytogenes. Appl. Environ. Microbiol. 77, 7841-7845. https://doi.org/10.1128/AEM.05732-11 (2011).

28. Fraser, K. R., Sue, D., Wiedmann, M., Boor, K. \& O’Byrne, C. P. Role of sigmaB in regulating the compatible solute uptake systems of Listeria monocytogenes: osmotic induction of opuC is sigmaB dependent. Appl. Environ. Microbiol. 69, 2015-2022. https://doi. org/10.1128/aem.69.4.2015-2022.2003 (2003).

29. Utratna, M., Cosgrave, E., Baustian, C., Ceredig, R. \& O’Byrne, C. Development and optimization of an EGFP-based reporter for measuring the general stress response in Listeria monocytogenes. Bioeng. Bugs 3, 93-103. https://doi.org/10.4161/bbug.19476 (2012).

30. D'Donoghue, B. A Molecular Genetic Investigation into Stress Sensing in the Food-Borne Pathogen Listeria monocytogenes: Roles for RsbR and Its Paralogues (National University of Ireland, Galway, 2016).

31. Becker, L. A., Cetin, M. S., Hutkins, R. W. \& Benson, A. K. Identification of the gene encoding the alternative sigma factor sigmaB from Listeria monocytogenes and its role in osmotolerance. J. Bacteriol. 180, 4547-4554 (1998).

32. Misra, S. K. et al. Analysis of the serine/threonine/tyrosine phosphoproteome of the pathogenic bacterium Listeria monocytogenes reveals phosphorylated proteins related to virulence. Proteomics 11, 4155-4165. https://doi.org/10.1002/pmic.201100259 (2011).

33. He, K. et al. Phosphorylation residue T175 in RsbR protein is required for efficient induction of sigma B factor and survival of Listeria monocytogenes under acidic stress. J. Zhejiang Univ. Sci. B 20, 660-669. https://doi.org/10.1631/jzus.B1800551 (2019).

34. Chan, Y. C., Boor, K. J. \& Wiedmann, M. SigmaB-dependent and sigmaB-independent mechanisms contribute to transcription of Listeria monocytogenes cold stress genes during cold shock and cold growth. Appl. Environ. Microbiol. 73, 6019-6029. https:// doi.org/10.1128/AEM.00714-07 (2007).

35. Chen, C. C., Lewis, R. J., Harris, R., Yudkin, M. D. \& Delumeau, O. A supramolecular complex in the environmental stress signalling pathway of Bacillus subtilis. Mol. Microbiol. 49, 1657-1669. https://doi.org/10.1046/j.1365-2958.2003.03663.x (2003).

36. Ondrusch, N. \& Kreft, J. Blue and red light modulates SigB-dependent gene transcription, swimming motility and invasiveness in Listeria monocytogenes. PLoS ONE 6, e16151. https://doi.org/10.1371/journal.pone.0016151 (2011).

37. Tiensuu, T., Andersson, C., Ryden, P. \& Johansson, J. Cycles of light and dark co-ordinate reversible colony differentiation in Listeria monocytogenes. Mol. Microbiol. 87, 909-924. https://doi.org/10.1111/mmi.12140 (2013).

38. O'Donoghue, B. et al. Blue-light inhibition of Listeria monocytogenes growth is mediated by reactive oxygen species and is influenced by sigmaB and the blue-light sensor Lmo0799. Appl. Environ. Microbiol. 82, 4017-4027. https://doi.org/10.1128/AEM.00685 -16 (2016).

39. Patange, A. et al. The effect of atmospheric cold plasma on bacterial stress responses and virulence using Listeria monocytogenes knockout mutants. Front. Microbiol. 10, 2841. https://doi.org/10.3389/fmicb.2019.02841 (2019).

40. Jia, X., Wang, J. B., Rivera, S., Duong, D. \& Weinert, E. E. An O2-sensing stressosome from a Gram-negative bacterium. Nat. Commun. 7, 12381. https://doi.org/10.1038/ncomms12381 (2016).

41. Monk, I. R., Gahan, C. G. \& Hill, C. Tools for functional postgenomic analysis of listeria monocytogenes. Appl. Environ. Microbiol. 74, 3921-3934. https://doi.org/10.1128/AEM.00314-08 (2008).

42. Jonquieres, R., Bierne, H., Fiedler, F., Gounon, P. \& Cossart, P. Interaction between the protein InlB of Listeria monocytogenes and lipoteichoic acid: a novel mechanism of protein association at the surface of gram-positive bacteria. Mol. Microbiol. 34, 902-914. https://doi.org/10.1046/j.1365-2958.1999.01652.x (1999).

\section{Acknowledgements}

We thank Juan C. Alonso (CNB-CSIC) for the B. subtilis strain BG214; Daniel López (CNB-CSIC) for the anti-GroEL antibody; Alberto Paradela, from the Proteomics Facility at CNB-CSIC, for his help in the interactome studies; and, Sara García-Escudero (Cytometry Unit, CNB-CSIC) for her help to use the Kaluza software. We thank Prof Rick Lewis (formerly University of Newcastle, UK) for helpful discussions about the design of mutant constructs. This work was supported by funds from the European Union's Horizon 2020 Research-andInnovation Program under Marie Sklodowska-Curie Grant Agreement No. 721456 (to C.O'B. and F.G-d.P.) and grant no. PGC2018-096364-B-I00 from the Spanish Ministry of Science and Innovation (to M.G.P.). Charlotte Dessaux and Duarte Guerreiro are supported by the Horizon 2020 Research-and-Innovation Program under Marie Sklodowska-Curie grant agreement no. 721456 as Early Stage Researchers.

\section{Author contributions}

C.D. and F.G.-d.P conceived and designed the experiments for this study. C.D. performed the experiments and collected the data. D.N.G. generated some of bacterial mutants used. C.D., M.G.P., C.O’B. and F.G.-d.P discussed 
and interpreted the data. The first draft was written by F.G.-d.P, which was critically commented by C.D., M.G.P. and C.O'B. All authors revised and approved the final version of the manuscript.

\section{Competing interests}

The authors declare no competing interests.

\section{Additional information}

Supplementary information is available for this paper at https://doi.org/10.1038/s41598-020-77738-z.

Correspondence and requests for materials should be addressed to F.G.P.

Reprints and permissions information is available at www.nature.com/reprints.

Publisher's note Springer Nature remains neutral with regard to jurisdictional claims in published maps and institutional affiliations.

(c) (i) Open Access This article is licensed under a Creative Commons Attribution 4.0 International License, which permits use, sharing, adaptation, distribution and reproduction in any medium or format, as long as you give appropriate credit to the original author(s) and the source, provide a link to the Creative Commons licence, and indicate if changes were made. The images or other third party material in this article are included in the article's Creative Commons licence, unless indicated otherwise in a credit line to the material. If material is not included in the article's Creative Commons licence and your intended use is not permitted by statutory regulation or exceeds the permitted use, you will need to obtain permission directly from the copyright holder. To view a copy of this licence, visit http://creativecommons.org/licenses/by/4.0/.

(C) The Author(s) 2020 